\title{
The dynamical evolution of massive black hole binaries - I. Hardening in a fixed stellar background.
}

\author{
Gerald D. Quinlan \\ Lick Observatory, University of California, Santa Cruz CA 95060 \\ Dept. of Physics and Astronomy, Rutgers University, PO Box 849, Piscataway NJ $08855^{*}$ \\ ${ }^{*}$ Present address
}

17 January 1996

\begin{abstract}
The stellar ejection rate and the rates of change of the binary semimajor axis and eccentricity are derived from scattering experiments for the restricted three-body problem. They are used to study the evolution of binaries in simple models for galactic nuclei, starting soon after the black holes become bound and continuing until the evolution is dominated by the emission of gravitational radiation, or until the ejected mass is too large for the galaxy to be considered fixed. The eccentricity growth is found to be unimportant unless the binary forms with a large eccentricity. The scattering results are compared with predictions from Chandrasekhar's dynamical-friction formula and with previous work on the capture and scattering of comets by planetary systems. They suggest that a binary with masses $m_{1} \geq m_{2}$ should not be considered hard until its orbital velocity exceeds the background velocity dispersion by a factor that scales as $\left(1+m_{1} / m_{2}\right)^{1 / 2}$.
\end{abstract}

\section{INTRODUCTION}

The existence of massive black hole $(\mathrm{BH})$ binaries follows from two widely-accepted assumptions: that galaxies merge with other galaxies, and that many galaxies contain massive BHs. For if two BHs enter the core of a merged galaxy, dynamical friction drags them to the center where they form a binary. The subsequent evolution was first outlined by Begelman, Blandford, and Rees (1980, hereafter BBR). Initially the binary hardens (i.e. its separation shrinks) because of the interaction between the BHs and all the stars in the galaxy core. But that is ineffective once the BHs become close because distant stars perturb the binary's center of mass but not its semimajor axis. The binary then hardens by giving kinetic energy to stars that pass in its immediate vicinity; a hard binary can eject stars out of the core at high velocity. If there are enough stars for the hardening to continue (and gas accretion onto the BHs can help), eventually the BHs merge through the emission of gravitational radiation; otherwise the hardening stalls and the binary survives for the lifetime of the galaxy.

Whether a binary merges or survives and how long it spends in each stage of the evolution are questions relevant to a number of problems in extragalactic astronomy. Their answers would help us predict the total $\mathrm{BH}$ merger rate and whether it is high enough for us to detect the resulting gravitational waves (e.g. Thorne 1992, Haehnelt 1994). They would help us assess BH-binary models for the bending and apparent precession of radio jets from active galactic nuclei, first proposed by BBR. And they would tell us what to expect if three or more BHs enter the core of a galaxy, which can happen if the BHs are dragged in from the galaxy's halo or if the galaxy undergoes multiple mergers with other galaxies containing BHs. If the first binary merges fast it can form a binary with a third $\mathrm{BH}$, and once that merges it can form a binary with a fourth, and so on, leading to a massive central $\mathrm{BH}$; but if the first binary still exists when a third $\mathrm{BH}$ enters then one or all three of the $\mathrm{BHs}$ can be ejected in a sling-shot interaction. Arguments like these can set limits on massive $\mathrm{BHs}$ as dark-matter candidates for galactic halos (see Hut and Rees 1992, Xu and Ostriker 1994 for conflicting limits for our Galaxy).

Another question is what a binary merger does to the surrounding galaxy, i.e. what observable signature it leaves. Mass ejection during the evolution should reduce a galaxy's central density and expand its core (BBR). Ebisuzaki, Makino, and Okumura (1991) have proposed this as an explanation for why large elliptical galaxies have lower central densities and weaker density cusps than small ellipticals (e.g. Kormendy et al. 1994).

We are far from having precise answers to any of these questions. BBR gave a range of merger times for one typical example that spanned three orders of magnitude because of the uncertain influence that mass ejection has on the hardening rate. Fukushige, Ebisuzaki and Makino (1992) have argued that dynamical friction causes a binary to become highly eccentric and that this greatly reduces the merger time because gravitational radiation then becomes impor- 
tant early in the evolution. Although their arguments are not convincing, they have called attention to the eccentricity growth and our ignorance of its correct description. There are uncertainties in how the hardening rate depends on the ratio of the two BH masses, in when a binary makes the transition from soft to hard, and even in what the words soft and hard should mean in this context. And our knowledge of how a binary merger changes a galaxy is based on back-of-the-envelope estimates and simple N-body experiments with unrealistic galaxy models.

The first step towards resolving these questions is to understand how a massive binary evolves in fixed stellar background. Consider a binary with masses $m_{1} \geq m_{2}$ and semimajor axis $a$ in an isotropic background of stars of mass $m_{*} \ll m_{2}$, density $\rho$, and one-dimensional velocity dispersion $\sigma$; let $M_{12}$ and $\mu$ denote the total and reduced binary mass:

$M_{12}=m_{1}+m_{2}, \quad \mu=m_{1} m_{2} / M_{12}$.

The binary evolution and its effect on the galaxy are determined by three dimensionless quantities: the hardening rate

$H=\frac{\sigma}{G \rho} \frac{d}{d t}\left(\frac{1}{a}\right)$,

the mass ejection rate (where $M_{\mathrm{ej}}$ is the stellar mass that the binary has ejected from the galaxy core)

$J=\frac{1}{M_{12}} \frac{d M_{\mathrm{ej}}}{d \ln (1 / a)}$,

and the eccentricity growth rate

$K=\frac{d e}{d \ln (1 / a)}$.

The quantities $H, J$, and $K$ can be found from scattering experiments that treat the star-binary encounters one at a time. Analytic approximations such as the impulse approximation are helpful during the early stages of the evolution (Gould 1991), but not once the binary becomes hard.

Most published scattering experiments assume the binaries and stars to have equal or nearly equal masses (see Heggie 1988 for a review). There are some exceptions. Roos (1981) performed scattering experiments for the restricted three-body problem to study the evolution of hard, massive $\mathrm{BH}$ binaries, and used them to correct a misplaced factor of $m_{1} / m_{2}$ in the BBR hardening rate. He tried to measure $K$ but his statistics were too poor to give definite conclusions (only 500 orbits per measurement). Hills (1983a) used the general three-body problem to study interactions between a massive binary and low-mass intruders $\left(m_{*} / m_{2}=0.01\right)$. He gave results for the hardening rate for a wide range of mass ratios $\left(m_{1} / m_{2}=1-300\right)$, but like Roos he considered only very hard binaries. Mikkola and Valtonen (1992) used the restricted three-body problem to measure $H$ and $K$ for equal-mass $\mathrm{BH}$ binaries with varying degrees of hardness. Their measurements are accurate for hard binaries but have large error bars for binaries that are not hard.

If $m_{1} \gg m_{2}$ then the interaction between a star and a $\mathrm{BH}$ binary is similar to the interaction between a comet and a planet orbiting a star. Although scattering experiments are used to study cometary dynamics (see Fernandez 1993 for a review) they are not of much help for our questions about $\mathrm{BH}$ binaries, partly because they often consider only one mass ratio (for the Sun-Jupiter system), but mostly because they are used to answer different questions, such as the cross section for the capture of interstellar comets, or for the conversion of long-period comets to short-period comets, the survival probability of comets once they are captured, and how all these depend on the comet's inclination. There is nevertheless some overlap between the two problems.

The goal of this paper is to present accurate measurements of $H, J$, and $K$ over the range of parameters of interest for the BH-binary problem, including the dependence on the mass ratio, eccentricity, and degree of hardness of the binary. Other quantities to be studied include the cross section for a binary to capture stars into bound orbits, for close encounters between stars and the binary members, and the distribution of velocities with which stars are expelled from the binary. These add to our understanding of $H, J$, and $K$, and are needed by themselves for some applications. The results will be presented in a model-independent way so that they can be applied to any problem with $m_{*} \ll m_{2} \leq m_{1}$.

Once $H, J$, and $K$ have been measured they can be used to study the evolution of binaries in fixed galaxy models. That will be done here for some simple models. If the $\mathrm{BHs}$ are large they will of course eject too much mass from the galaxy core for it to be considered fixed. But the results will still be valid during the early stages of the evolution. And they will be helpful even in the later stages, because we can imagine at any instant that the binary is embedded in a fixed background whose properties are those of the galaxy at that instant. The self-consistent evolution of a massive binary in a realistic galaxy model and the changes this induces in the model are best studied by large N-body experiments. That will be deferred to paper II, along with a discussion of what both papers imply for the astronomical questions mentioned above (Quinlan and Hernquist, in preparation).

\section{COMPUTATIONAL METHOD}

\subsection{Derivation of results from the restricted three-body problem}

We treat the star as a massless test particle moving in the potential of the two BHs. From the changes in the star's energy and angular momentum per unit mass, $\Delta E_{*}$ and $\Delta L_{*}$, we infer the corresponding changes $\Delta E$ and $\Delta L$ that the binary would have suffered if the star had been given a small but nonzero mass. The three bodies are treated as point masses and gravitational radiation is ignored.

In a real galaxy stars approach the binary with a wide distribution of velocities at any given time. But the scattering experiments are easiest to perform if the stars all start from the same velocity $v$ at a large separation from the binary (the initial velocity, or the velocity at infinity). In that case we write 
$\frac{d}{d t}\left(\frac{1}{a}\right)=\frac{G \rho}{v} H_{1}$,

where the "1" indicates that the stars all have a single velocity $v$. The hardening rate for a Maxwellian velocity distribution is then

$H(\sigma)=\int_{0}^{\infty} d v 4 \pi v^{2} f(v, \sigma) \frac{\sigma}{v} H_{1}(v)$,

where

$f(v, \sigma)=\frac{1}{\left(2 \pi \sigma^{2}\right)^{3 / 2}} \exp \left(-v^{2} / 2 \sigma^{2}\right)$.

The hardening rate is derived from the average energy change for stars that scatter off the binary. We define a dimensionless energy change $C$ by (Hills 1983a)

$C=\frac{M_{12}}{2 m_{*}} \frac{\Delta E}{E}=\frac{a \Delta E_{*}}{G \mu}$.

This must be averaged over all angular variables describing the binary's orientation and phase, to give $\langle C\rangle$, and then integrated over all impact parameters. The averaging and integrating are done in a Monte Carlo fashion by picking orbits from suitable distributions. We sometimes describe an orbit by its impact parameter $b$, the distance at which it would pass the binary if it felt no attraction, and sometimes by its pericenter distance $r_{p}$, the distance if it is attracted by a point mass $M_{12}$. The two are related by gravitational focusing:

$b^{2}=r_{p}^{2}\left(1+\frac{2 G M_{12}}{r_{p} v^{2}}\right)$.

We also define a dimensionless impact parameter $x$ by

$x=b / b_{0}, \quad b_{0}^{2}=2 G M_{12} a / v^{2} ;$

$b_{0}$ is approximately the impact parameter corresponding to $r_{p}=a$ if gravitational focusing is important. With this notation we can write

$H_{1}=8 \pi I_{x}(C)=8 \pi \int_{0}^{\infty} d x x\langle C\rangle$,

where the second equality defines the operator $I_{x}$.

The derivation of the eccentricity growth rate is similar. The change to the binary's eccentricity from a single scattering event is, if the change is small and the binary's orbital angular momentum points in the $z$ direction,

$\frac{\Delta e}{\Delta \ln (1 / a)}=\frac{\left(1-e^{2}\right)}{2 e}\left[-2\left(\frac{\Delta L_{z}}{L_{z}}\right)\left(\frac{E}{\Delta E}\right)-1\right]$.

We define a dimensionless angular-momentum change $B$ by

$B=-\frac{M_{12}}{m_{*}} \frac{\Delta L_{z}}{L_{z}}=\frac{M_{12}}{\mu} \frac{\Delta L_{*, z}}{\left[G M_{12} a\left(1-e^{2}\right)\right]^{1 / 2}}$,

and can then write

$K_{1}=\frac{\left(1-e^{2}\right)}{2 e}\left[\frac{I_{x}(B-C)}{I_{x}(C)}\right]$,

where the " 1 " has the same meaning as before. The derivation of $K$ from $K_{1}$ will be described later.

\subsection{The scattering experiments}

Each scattering experiment requires five uniformlydistributed random numbers (four if the binary is circular): one for the square of the impact parameter (in some range $\left.\left[0, b_{\max }^{2}\right]\right)$, and four to fix the binary's orientation and phase: the cosine of the inclination $([-1,1])$, the longitude of ascending node $([0,2 \pi])$, the argument of pericenter $([0,2 \pi])$, and the mean anomaly at some fixed time $([0,2 \pi])$. The numbers are chosen with the quasi-random number generator sobseq of Press et al. (1992).

The range $\left[0, b_{\max }\right]$ for impact parameters is split into five intervals corresponding to ranges in scaled pericenter distance $r_{p} / a$ of $[0,1],[1,2],[2,4],[4,8]$, and $[8,16]$. Each output quantity is measured in a number of steps. On the first step the program spends short but equal amounts of cpu time picking orbits from the five intervals. On each successive step the program doubles the cpu time and adjusts its strategy so that the time it spends on each interval is proportional to the uncertainty that interval contributes to the quantity being measured. Once the uncertainty is reduced to an acceptable level, or the cpu time exceeds some maximum allowed value, the results from all five intervals are combined with appropriate weights for a distribution uniform in $b^{2}$. For $H, J$, and $K$ the last three intervals contribute little because the changes in energy and angular momentum fall off rapidly with increasing impact parameter.

The coordinates are chosen so that the binary's center of mass is at the origin and the star starts at infinity with $(x, y, z)=(b, 0, \infty)$ and $\left(v_{x}, v_{y}, v_{z}\right)=(0,0,-v)$. The star is moved from $r=\infty$ to $r=50 a$ along a Keplerian orbit about a point mass $M_{12}$ at the origin. The numerical integration starts at $r=50 a$.

The orbits are integrated in double precision with an explicit, embedded Runge-Kutta method of order (7)8: the program dopri8 of Hairer, Norsett, and Wanner (1987). The program adjusts the integration stepsize to keep the fractional error per step in the position and velocity below some level $\epsilon$, which was set to $10^{-9}$. With this choice the change in a star's Jacobi constant for a circular binary is at most $10^{-6} G M_{12} / a$ and often much smaller. The forces from the BHs are not softened.

Some integrations are time consuming because the star gets captured into a weakly-bound orbit and makes many revolutions before it is expelled. The integration stepsize is a small fraction of the binary's period even if the period of the star about the binary is much longer. The following approximation expedites those experiments. If a captured star moves further than $r_{k}=a\left(10^{10} m_{2} / m_{1}\right)^{1 / 4}$ from the binary, the binary is replaced by a point mass $M_{12}$ and the star is moved along a Keplerian ellipse until it returns inside $r_{k}$, when the forces from the BHs are reintroduced with the correct orbital phase. The $\left(m_{2} / m_{1}\right)^{1 / 4}$ mass scaling makes the quadrupole force from the binary at $r_{k}$ about $10^{-10} G M_{12} / a^{2}$, independent of $m_{2} / m_{1}$.

Orbits that get captured for long times tend to be highly chaotic. The integration for any particular orbit of that type is difficult to justify because a small change to the integration procedure can make a big change to the out- 
come. But the average results derived from a large number of integrations can be correct even if the individual integrations are not; that is suggested by shadowing lemmas that have been proved for simple chaotic systems. The average results presented here do not change noticeably if $\epsilon$ is raised or lowered by a factor of 100 , even though some orbits undergo big changes.

An integration is stopped when the star leaves the sphere $r=50 a$ with positive energy. The average results are not sensitive to the location of this sphere provided that it is at least $10-15$ times larger than $a$. Once the integration stops the program records the changes to the star's energy and angular momentum, the minimum separations between the star and the two BHs, and between the star and the binary's center of mass, the integration time, the number of integration steps, and the number of times the star's radius passed through a minimum (which, if greater than one, gives the number of revolutions that a captured orbit made). An orbit that does not get captured typically takes a few hundred to a few thousand integration steps. Captured orbits can take much longer. If an integration lasts for more than $10^{6}$ steps it is abandoned. The fraction of abandoned integrations is the largest for hard binaries with $m_{1} / m_{2} \gg 1$, but even for those it is less than $0.1 \%$.

The error in any average quantity has a systematic component and a statistical component. Systematic errors arise, for example, because of errors in the numerical integration, because integrations are abandoned if they take too long, because the program imposes a maximum impact parameter $b_{\max }$, and because the orbits start and end at $r=50 a$ instead of at $r=\infty$. But none of these is large: the total systematic error is usually much smaller than the statistical error. The statistical errors are estimated by taking the difference between results found with $N$ orbits (the final number) and $N / 2$ orbits, or sometimes - if that difference looks suspiciously small - one half the difference between $N$ orbits and $N / 4$ orbits. That gives a rough estimate of the error level. The statistical errors decrease at large $N$ as $(\ln N)^{d} / N$ when quasi-random numbers are used, where $d$ is the number of numbers picked for each experiment ( 5 in general, 4 if the binary is circular). That is faster than the $N^{-1 / 2}$ decrease that occurs with random numbers (Press et al. 1992).

The number of orbits needed to reduce the statistical errors to an acceptable level varies widely with the binary and the measurement. Measurements are more difficult for $K_{1}$ than for $H_{1}$ because of cancellation. Cancellation is a problem for $H_{1}$ too at high $v$ values. And regardless of what is being measured, binaries with $m_{1} \gg m_{2}$ require many more orbits than equal-mass binaries because of the rare close encounters with the mass $m_{2}$. When the results are presented the number of orbits used will not be given because it is different for each measurement; some estimate of the statistical error will be given instead. The numbers are about $10^{4}-10^{5}$ orbits per measurement for $H_{1}$ (sometimes more at high $v$ values) and $10^{5}-10^{6}$ for $K_{1}$. The complete set of experiments took about four months of cpu time on an IBM 580 RISC workstation.

\begin{tabular}{rrr}
\hline$m_{1} / m_{2}$ & $H_{0}$ & $w / V_{\text {bin }}$ \\
\hline 1 & 17.97 & 0.5675 \\
4 & 20.54 & 0.4263 \\
16 & 21.87 & 0.2228 \\
64 & 22.78 & 0.1043 \\
256 & 22.57 & 0.0573 \\
\hline
\end{tabular}

Table 1. Parameters for fits to $H_{1}$ (eq. 16) for a circular binary.

\section{RESULTS FROM THE SCATTERING EXPERIMENTS}

\subsection{Hardening}

\subsubsection{Hardening rate}

The hardening rate $H_{1}$ (5) has been measured as a function of the binary's eccentricity and hardness for a wide range of mass ratios. It is plotted in Figure 1 versus the hardness as given by the ratio of the initial stellar velocity $v$ and the binary's orbital velocity $V_{\text {bin }}$ (the relative velocity of the two BHs if the binary is circular):

$V_{\mathrm{bin}}=\sqrt{G M_{12} / a}$.

The error bars show the statistical-error estimates; if not visible then they are smaller than the size of the points.

The velocity dependence of $H_{1}$ is fit by a function with two free parameters (whose values are given in Table 11):

$H_{1}=\frac{H_{0}}{\left[1+(v / w)^{4}\right]^{1 / 2}}$.

The function has a constant value $H_{0}$ at $v \ll w$, starts to decrease as $v$ approaches $w$, and decreases as $1 / v^{2}$ at $v \gg w$. This fits the data well at high and low velocities. It does not fit so well when $H_{1}$ first starts to decrease; those data points were given little weight in the fitting procedure. A three-parameter function was also tried, $H_{1}=H_{0} /\left[1+(v / w)^{\kappa}\right]^{2 / \kappa}$, but the exponent $\kappa$ never differed by much from 4 .

For an equal-mass binary the constant hardening rate at low velocity, $H_{0} \simeq 18.0$, agrees well with Mikkola and Valtonen's (1992) $\pi R_{a} \simeq 18.2$, and also with the results of Hills $(1983 \mathrm{a}, 1992)$. At high velocity $H_{1} \approx$ $H_{0}(w / v)^{2} \simeq 5.8\left(V_{\mathrm{bin}} / v\right)^{2}$, which can be compared with Gould's (1991) analysis using the impulse approximation, $H_{1, \text { Gould }}=(8 \pi / 3)\left(V_{\mathrm{bin}} / v\right)^{2}$. The agreement is satisfactory considering that the error bars are large at high velocity and that the impulse approximation is justified only if $v / V_{\text {bin }} \gg 1$. In fact it is surprising how well the impulse approximation works when $v / V_{\mathrm{bin}} \simeq 1$.

Panel (a) shows that the hardening rate for an equalmass binary does not vary much with the eccentricity. Mikkola and Valtonen (1992) reached the same conclusion, which remains true for all mass ratios. For later applications the hardening rate for a circular binary will be used for all eccentricities because the variation of $H_{0}$ and $w$ with eccentricity is too small to matter. 

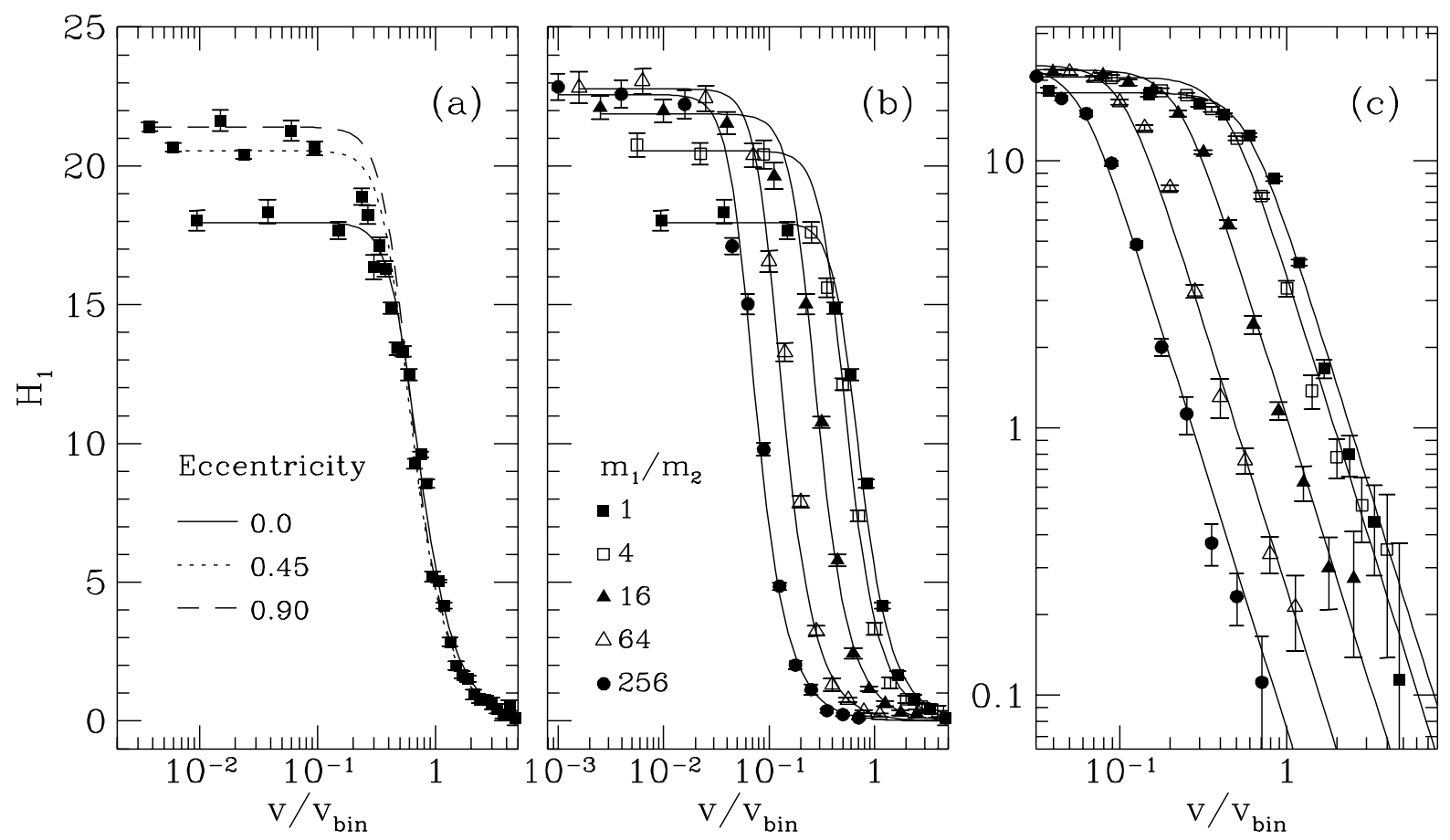

Figure 1. Binary hardening rate versus the initial stellar velocity $v$ : (a) shows three eccentricities for $m_{1} / m_{2}=1$; (b) and (c) show five mass ratios for $e=0$, on linear and logarithmic scales. The lines are the fits to eq. (16).

Roos (1981) and Hills (1983a) showed that the lowvelocity hardening rate $H_{0}$ does not vary much with the mass ratio. But the velocity $w$ does. The variation is fit by

$w \simeq 0.85 \sqrt{\frac{m_{2}}{M_{12}}} V_{\mathrm{bin}}=0.85 \sqrt{\frac{G m_{2}}{a}}$.

The physical significance of this mass dependence is the following: if $v<w$ the binary can easily capture stars into bound orbits; if $v>w$ it cannot.

The integral (6) for a Maxwellian distribution was evaluated numerically. The relation between $H$ and $H_{1}$ is fit closely by the formula

$\frac{H(\sigma)}{H_{1}(\sqrt{3} \sigma)} \simeq \sqrt{\frac{2}{\pi}}+\ln \left[1+\alpha\left(\frac{\sigma}{w}\right)^{\beta}\right]$,

with $\alpha=1.16$ and $\beta=2.40$. In the limit of high velocity this gives

$H \approx \frac{\beta H_{0}}{6}\left(\frac{w^{2}}{\sigma^{2}}\right) \ln \left(\frac{\sigma^{2}}{w^{2}}\right)$.

The log term looks like a familiar Coulomb logarithm but comes from an integral over the velocity distribution, not over a range of impact parameters. The limit (19) can be compared with Gould's (1991) hardening rate,

$H_{\text {Gould }}=\frac{16 \sqrt{2 \pi}}{3}\left(\frac{G \mu}{a \sigma^{2}}\right) \ln \left(\frac{\sigma^{2}}{V_{\mathrm{bin}}^{2}}\right)$.
For an equal-mass binary the coefficients of the log terms differ by about $30 \%$, which is satisfactory considering the uncertainties mentioned above. For non-equal masses Gould's log term does not have the correct mass dependence (Gould did not attempt to compute the log term accurately).

The hardening rate for a massive BH binary is sometimes derived from Chandrasekhar's dynamical-friction formula (e.g. Fukushige et al. 1992). The error in that has been known for many years (Chandrasekhar 1944, Hills 1983a): the distant encounters included in the friction formula do not perturb the binary's semimajor axis - they only perturb its center of mass. It is an accident that the derivation gives a result like Gould's for a Maxwellian distribution if a suitable choice is made for the log term: if the same derivation is used for $H_{1}$ it gives the nonsense result that (for $m_{1}=m_{2}$ ) $H_{1}$ is zero at $v=0$, rises as $H_{1} \sim v$ for $v<V_{\text {bin }} / 2$, and then drops abruptly back to zero at $v=V_{\text {bin }} / 2$ (because only stars moving slower than the BHs contribute in Chandrasekhar's formula). See Gould (1991) for further discussion.

The velocity dependence of the hardening rate suggests a new convention for the use of the word hard. A hard binary is usually defined in one of three ways. The first says that a binary with binding energy $E_{\mathrm{b}}$ is hard if $E_{\mathrm{b}} \gg m_{*} \sigma^{2}$ and soft if $E_{\mathrm{b}} \ll m_{*} \sigma^{2}$ (p. 534 of Binney and Tremaine 1987). The second says a binary is hard if it grows harder through interactions with stars and soft if it grows softer (Hut 1983). And the third, which is often 
stated as a corollary of the first or second rather than as an independent definition, says a hard binary is one that "hardens at a constant rate," i.e. at a rate $H$ that is independent of the hardness. The equivalence of the first two definitions is called Heggie's law. But neither of those definitions is useful for a massive BH binary because both are satisfied by almost any pair of massive $\mathrm{BHs}$ that is close enough to be called a binary.

The third definition gives almost the same result as the first two when the masses are equal (see Hut 1983, or Figure 6.3 of Spitzer 1987) and is far more useful when the binary is massive. A BH binary will therefore be called hard if it hardens at a constant rate, i.e. if $\sigma \ll w$ or equivalently if $V_{\text {bin }} / \sigma \gg\left(1+m_{1} / m_{2}\right)^{1 / 2}$. It is tempting to call a binary soft if it is not hard, but that is confusing for massive binaries because there is a wide gap for them between a hard binary in the sense used here and a soft binary in the familiar sense that "soft binaries grow softer." In later figures the properties of hard binaries are studied with scattering experiments using the lowest initial velocity $v$ in Figure 1 for each mass ratio; those velocities are $\log _{10}(v)=-2.025$, $-2.25,-2.6,-2.8$, and -3.0 for $m_{1} / m_{2}=1,4,16,64$, and 256.

The scattering results and Gould's (1991) analysis refute Hills's (1990) statement that a binary grows harder if $V_{\text {bin }}>\sigma$ and softer if $V_{\text {bin }}<\sigma$ regardless of the values of $m_{*}, m_{1}$, and $m_{2}$. Although the mean energy change $\langle C\rangle$ at zero impact parameter does change from positive to negative when the stellar velocity is raised from $v<V_{\text {bin }}$ to $v>V_{\mathrm{bin}}$, that sign change disappears when $\langle C\rangle$ is averaged over impact parameter.

The reason for the hard/not-hard transition at $\sigma=w$ is best explained after we have examined the cross section for stars to be captured by a binary, to have close encounters with the binary members, and the distribution of velocities with which stars are expelled from a binary.

\subsubsection{Capture cross section}

We say that a binary captures an incoming star if the star's orbital radius passes through more than one minimum. Almost all captured orbits are eventually expelled in the three-body problem (there might be a set of measure zero that remain bound forever), but the star can survive for many revolutions before that happens.

Previous work has used scattering experiments and approximate methods to derive capture cross sections. Hills has used scattering experiments to study the capture of orbits by very hard, massive binaries (Hills 1983a, 1983b, 1992). He unfortunately defines capture - or what he calls long-term capture - in a way that depends on his program (he says a long-term capture occurs if the integration takes more than 150,000 steps). But he gives helpful information on how the capture probability depends on the impact parameter, eccentricity, and binary mass ratio. Pineault and Duquet (1993) have used the impulse approximation to derive approximate capture cross sections for massive, circular binaries, for arbitrary mass ratios and degrees of hardness (they give many relevant references to the comet

\begin{tabular}{rrrrrr}
\hline$m_{1} / m_{2}$ & $c_{1}$ & $c_{2}$ & $c_{3}$ & $c_{4}$ & $c_{5}$ \\
\hline 1 & 17.97 & 1.0066 & 3.5745 & 2.0865 & 0.6100 \\
4 & 20.54 & 0.7929 & 4.5326 & 1.2675 & 1.2377 \\
16 & 21.87 & 0.4122 & 3.6588 & 1.2324 & 0.9754 \\
64 & 22.78 & 0.1800 & 6.1855 & 0.5562 & 1.0087 \\
256 & 22.57 & 0.0846 & 8.1992 & 0.3856 & 0.9782 \\
\hline
\end{tabular}

Table 2. Parameters for fits to $\Sigma_{\text {cap }}$ (eq. 22 for a circular binary.

literature). They say their cross sections are accurate to within a factor of $2-3$, although that is not clear because they adjust their formulas in an ad hoc way - using Hills's (1983a) results to guide them - for hard binaries for which the impulse approximation does not work.

The measurements made here improve upon those of Hills by using a reproducible capture definition and by exploring the dependence on the binary's degree of hardness. Panels (a) and (b) of Figure 2 show the capture cross section for a circular binary in units of the binary's geometrical cross section $\Sigma_{\text {bin }}$, which includes the correction for gravitational focusing:

$\Sigma_{\text {bin }}=\pi a^{2}\left(1+\frac{2 G M_{12}}{a v^{2}}\right)$.

The velocity dependence is fit by the function

$\frac{\Sigma_{\text {cap }}}{\Sigma_{\text {bin }}}=c_{1}\left[1+\left(\frac{v}{c_{2} V_{\text {bin }}}\right)^{c_{3}}\right]^{-c_{4}} \ln \left[1+\left(\frac{c_{2} V_{\text {bin }}}{v}\right)^{c_{5}}\right]$;

the five parameters are listed in Table日 (the fits should not be extrapolated to velocities much higher than shown in the figure). At low velocity $\Sigma_{\text {cap }} / \Sigma_{\text {bin }}$ rises as $\ln (1 / v)$ because the energy change $C$ decreases exponentially with impact parameter. At high velocity $\Sigma_{\text {cap }} / \Sigma_{\text {bin }}$ decreases as a power of $v$, which is clearer for the binaries with $m_{1} / m_{2} \gg 1$. The velocity at the transition between the logarithmic and power-law behavior, approximately $c_{2} V_{\text {hin }}$, depends on the mass ratio in the same way as $w$ (eq. 17).

For most velocities and mass ratios the cross sections in Figure 2 agree to within a factor of two with the approximate cross sections of Pineault and Duquet (1993). There are some larger differences at high velocity for an equalmass binary, and at $v / V_{\text {bin }} \simeq 0.01-0.1$ when $m_{1} / m_{2} \gg 1$. The $\left(v / V_{\mathrm{bin}}\right)^{-4}$ behavior seen when $m_{1} / m_{2} \gg 1$ also agrees with that found by Pineault and Duquet.

The capture cross section rises with the binary eccentricity, but the dependence is weak. The difference in $\Sigma_{\text {cap }}$ for circular and highly-eccentric binaries is only $20-30 \%$, too small to matter for most applications.

Panels (c) and (d) of Figure 2 show the cross section for a captured orbit to survive for at least $N$ revolutions, i.e. for the radius to pass through at least $N+1$ minima before the star is expelled. The results for binaries with $m_{1} / m_{2} \gg 1$ are fit well by $\Sigma_{\text {cap }}(N) / \Sigma_{\text {cap }} \sim N^{-1 / 2}$. Everhart (1976) noticed this $N^{-1 / 2}$ scaling in the survival of comets scattered by the Sun-Jupiter system, and interpreted it as resulting from a random-walk in the comet's energy, as in the gambler's ruin problem from probability theory (see Yabushita 1979, Quinn, Tremaine, and Duncan 

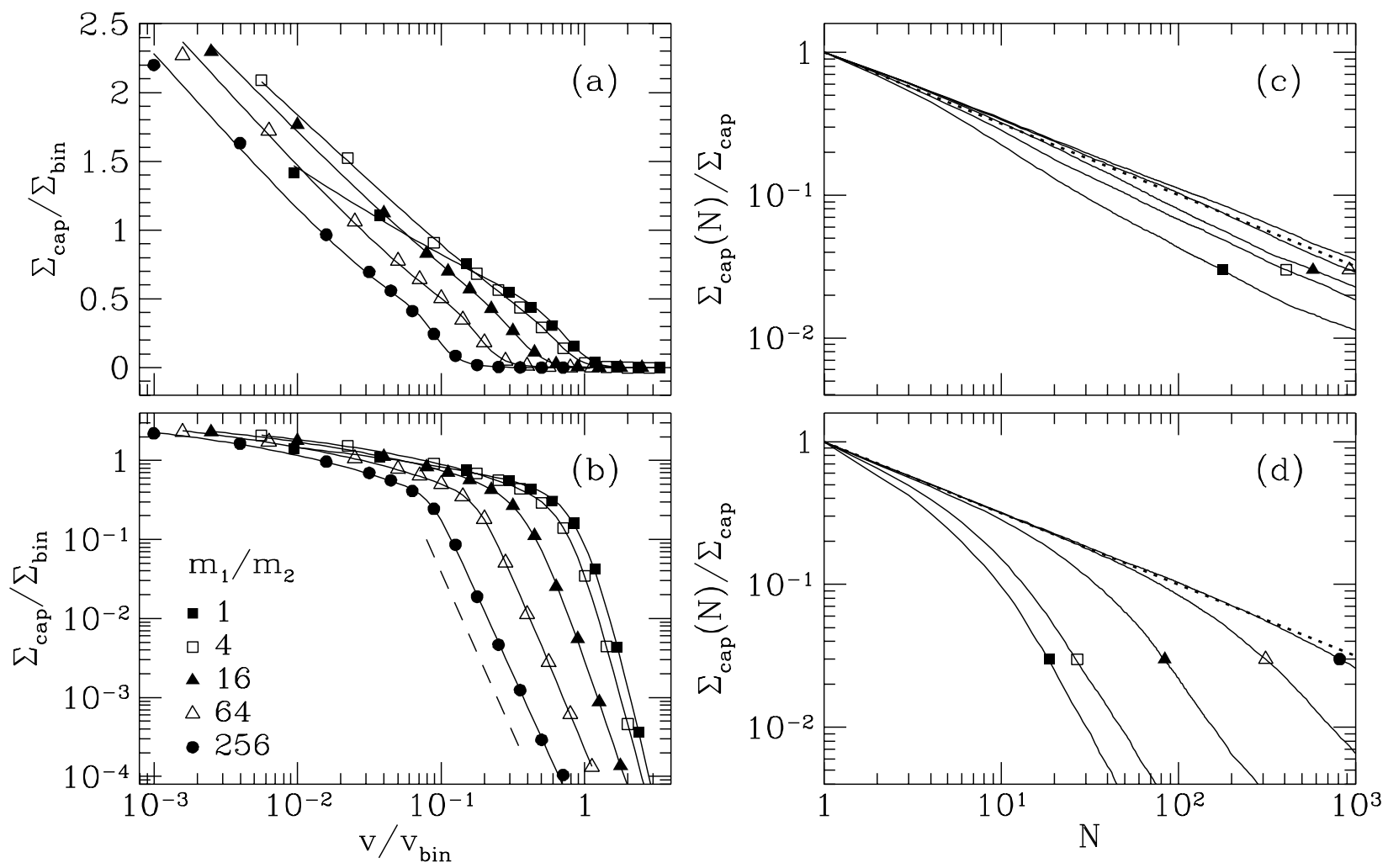

Figure 2. The cross-section $\Sigma_{\text {cap }}$ for a circular binary to capture an orbit with initial velocity $v$, plotted for five mass ratios on (a) linear and (b) logarithmic scales. The solid lines in (a) and (b) are the fits to eq. 222); the dashed line varies as $\left(v / V_{\text {bin }}\right)^{-4}$. Panels (c) and (d) show the cross section for an orbit to be captured for at least $N$ revolutions for the same five mass ratios, plotted in (c) at the lowest $v$ for each mass ratio, and in (d) at $v=w$. The dotted lines in (c) and (d) vary as $N^{-1 / 2}$.

1990 for further discussion). The $N^{-1 / 2}$ scaling does not work as well if the binary is not hard or if $m_{1} \simeq m_{2}$.

The cross sections in Figure 2 place no limit on the apocenter of the captured orbit. Some of the stars contributing to $\Sigma_{\text {cap }}$ are captured into weakly-bound orbits with apocenters many orders of magnitude larger than the binary's semimajor axis. In a real galaxy those orbits will be perturbed by passing stars and the galactic potential before they return to the binary. But that should not change the hardening rate much because the contribution from weaklybound captures is small, even when $m_{1} \gg m_{2}$.

\subsubsection{Close-encounter cross section}

The cross section for close encounters with the binary members is needed for applications to real problems where the bodies are not point masses. For a massive BH binary we need it to compute the rate at which stars are tidally disrupted by the BHs, and to estimate how those disruptions might change the hardening rate.

Figure $3(\mathrm{a})$ shows the cross section $\Sigma$ for a star to approach within a distance $\leq r$ of either of the BHs, for a hard, circular binary with $m_{1} / m_{2}=64$. The cross section is plotted for two sets of experiments: in the first the stars were allowed to encounter the binary only once, even if they were captured; the second allowed as many encounters as necessary for the stars to be expelled.

The cross section for the larger BH scales as $\Sigma / \Sigma_{\text {bin }} \sim$ $r$ for the single-encounter experiments because of gravitational focusing. For the multiple-encounter experiments $\Sigma$ is larger but the increase is important only for $r / a<$ $m_{2} / m_{1}$. The reason the increase is unimportant for $r / a>$ $m_{2} / m_{1}$ is that when a captured star orbits a binary with $m_{1} \gg m_{2}$ the star's distance of closest approach to $m_{1}$ remains nearly constant while its energy undergoes (approximately) a random walk. This is well known in cometary dynamics, where comets diffuse in energy at nearly constant perihelion (e.g. Duncan, Quinn, and Tremaine 1987).

The cross section for a close encounter with the smaller $\mathrm{BH}$ is different. For the single-encounter experiments gravitational focusing is important for $r / a<m_{2} / m_{1}$ but not for $r / a>m_{2} / m_{1}$, so $\Sigma / \Sigma_{\text {bin }}$ scales as $r$ or as $r^{2}$ depending on whether $r / a$ is smaller or larger than $m_{2} / m_{1}$. For the multiple-encounter experiments the cross section is larger for all values of $r$, not just for $r / a<m_{2} / m_{1}$.

The distance $r / a=m_{2} / m_{1}$ has a special importance for $m_{2}$ if $m_{1} \gg m_{2}$ because the velocity of a star orbiting $m_{2}$ at that distance equals $V_{\text {bin. }}$. Figure $3($ b) shows the cross section $\Sigma$ for such encounters as a function of the mass ratio. For the single-encounter experiments $\Sigma / \Sigma_{\text {bin }} \sim$ 

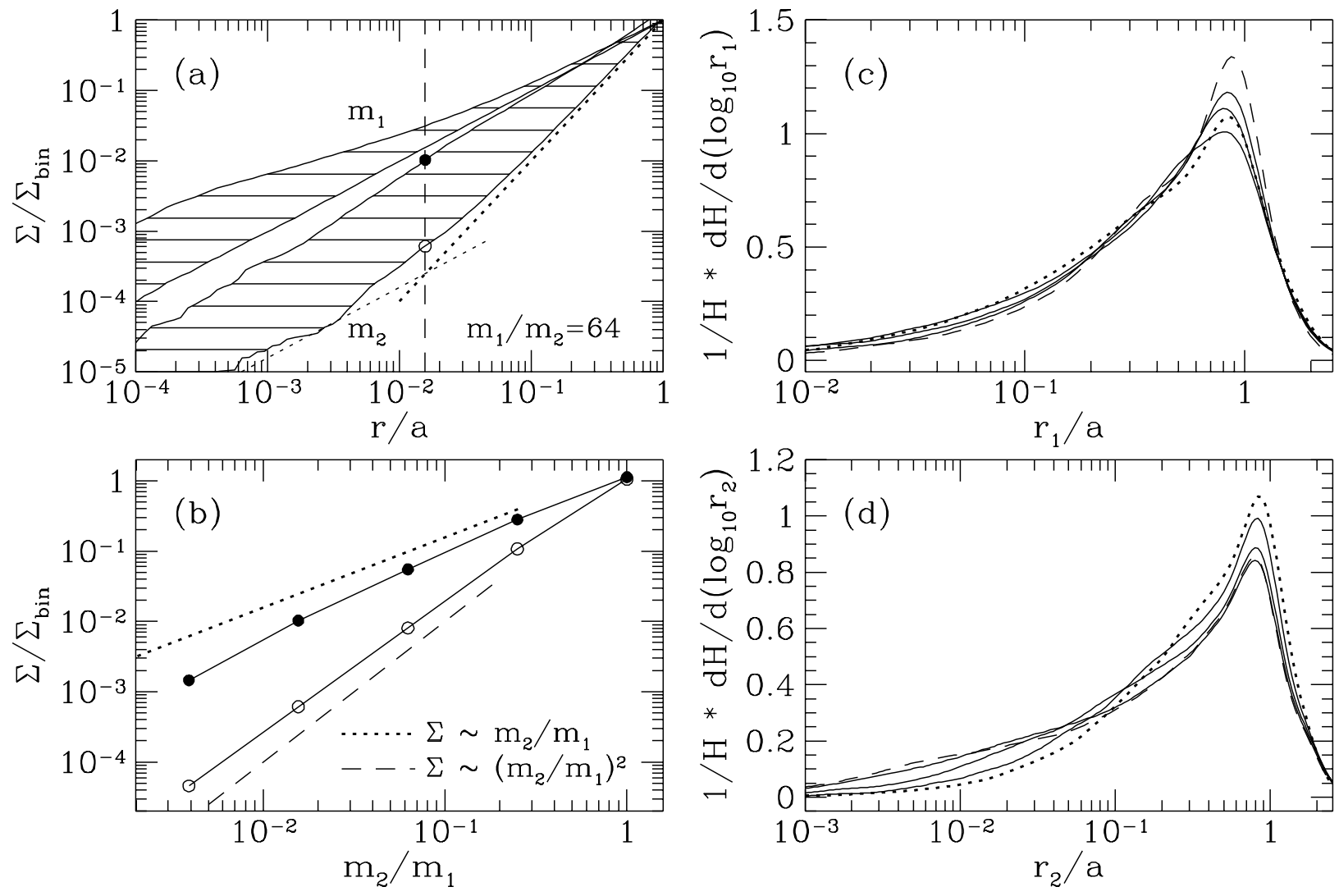

Figure 3. Close-encounter cross sections for a hard, circular binary ( $v$ equals the lowest value in Fig. 1 for each mass ratio). Panel (a) shows the cross section for an orbit to approach within $\leq r$ of $m_{1}$ or $m_{2}$. The lines bounding the upper shaded region are for $m_{1}$ : the lower line results when the orbits encounter the binary only once; the upper when they have as many encounters as necessary for them to leave with positive energy. The lower shaded region has the same meaning, but for $m_{2}$. The dotted lines vary as $r^{-1}$ and $r^{-2}$; the dashed line is at $r / a=m_{2} / m_{1}$. Panel (b) shows the cross section for an orbit to approach within $r \leq\left(m_{2} / m_{1}\right) a$ of $m_{2}$, for both single encounters (open circles) and multiple encounters (filled circles). Panels (c) and (d) show the differential hardening rates with respect to the distances of closest approach to $m_{1}$ and $m_{2}$; the five lines are for $m_{1} / m_{2}=1$ (dotted), 4, 16, 64, and 256 (dashed).

$\left(m_{2} / m_{1}\right)^{2}$; for the multiple-encounter experiments captures raise that scaling almost to $\Sigma / \Sigma_{\text {bin }} \sim m_{2} / m_{1}$.

Panels (c) and (d) of Figure 3 show the differential hardening rates with respect to the distances of closest approach to $m_{1}$ and $m_{2}$, normalized so that the area under the curves is unity. The largest contribution to the hardening comes from orbits that pass both BHs at a distance not much smaller than the semimajor axis. When $m_{1} \gg m_{2}$ there is a wide tail in the left of panel (d), but the contribution from close encounters with $m_{2}$ is still a small fraction of the total hardening rate.

In a real galaxy there will be two complications that can change these results. If weakly-bound captured stars are perturbed by nearby stars or the galactic potential they will not return to the binary in such a way as to keep their distance of closest approach to $m_{1}$ nearly constant. That would increase the difference between the single- and multiple-encounter cross sections for $m_{1}$. But if the captured stars are perturbed too much they might not return at all, which would reduce the cross sections for both $m_{1}$ and $m_{2}$. The two complications tend to cancel for $m_{1}$.

\subsubsection{Distribution of final velocities}

The final velocity is the velocity of a star at infinity after it has been expelled by the binary. We need their distribution to compute the mass ejection rate.

Everhart's $(1968,1969)$ work on the scattering of comets by planetary systems is relevant to the distributions to be considered here. Everhart used an approximate conicmatching procedure to derive the probability $h(U) d U$ for the energy change $U=\Delta E_{*}$ to lie in the interval $d U$ after a single encounter between the comet and the planet. The distribution has three parts, which Everhart called A, B, and C. Parts A and B are for the small and intermediate energy changes and are fit well by (A) a Gaussian and (B) $h(U) \sim 1 /|U|^{3}$. Part $\mathrm{C}$ is for the large energy changes resulting from rare, close encounters with the planet. In parts A and $\mathrm{B}, h(U)$ depends only on $|U|$, but that symmetry is 
broken in part $\mathrm{C}$ where energy gains are more frequent than energy losses.

Panel (a) of Figure 4 shows the final-velocity distribution for a hard binary from scattering experiments done in a manner similar to Everhart's, so that "final" means after a single encounter with the binary. If a star was captured its final velocity was set to $-\sqrt{2\left|E_{*}\right|}$, with $E_{*}$ measured when the star began returning to the binary for a second encounter; otherwise the final velocity was set to $\sqrt{2 E_{*}}$ at the end of the integration. The figure shows the cross sections $\Sigma$ for the final velocity to be greater than $v_{f}$ or less than $-v_{f}$ for some positive $v_{f}$.

For the binaries with $m_{1} / m_{2} \gg 1$ there is a range of velocities for which $\Sigma$ is symmetric (depends only on $\left|v_{\mathrm{f}}\right|$ ) and varies as $\Sigma \sim 1 / v_{f}^{4}$. This corresponds to Everhart's part B. The hardening rate would be nearly zero for these binaries if multiple encounters were not allowed because the positive and negative contributions would nearly cancel. The symmetry is not as good for binaries with equal masses for which the star is more likely to gain energy. That is why the $N^{-1 / 2}$ scaling in Figure 1 did not work so well for equal-mass binaries. The asymmetry that Everhart predicted for part $\mathrm{C}$ is not clear in the figure, perhaps because the statistics are poor when $m_{1} \gg m_{2}$ for the rare, close encounters with the mass $m_{2}$ (the $m_{1} / m_{2}=256$ results come from $10^{6}$ orbits, but that is still not enough).

Panel (b) shows the final-velocity distribution when the stars are allowed to encounter the binary as many times as necessary for them to be expelled. The $\Sigma \sim 1 / v_{f}^{4}$ scaling from the single-encounter experiments is raised to approximately $\Sigma \sim 1 / v_{f}^{3}$, but the probability of a star being expelled with $v_{f} \simeq V_{\text {bin }}$ is still small if $m_{1} \gg m_{2}$.

Panel (c) shows the differential hardening rate with respect to the final velocity, normalized so that the area under the curves is unity. The velocity at the maximum scales with the mass ratio in the same way as $w$ (eq. 17) and is approximately $1.75 \mathrm{w}$. There is a wide tail to the right of the maximum if $m_{1} \gg m_{2}$ but the high velocities contribute little to the hardening. In fact the hardening rate for $m_{1} \gg m_{2}$ can be computed quite accurately by considering just the positive velocities from panel (a) and multiplying the result by two, i.e. by assuming that the captured orbits eventually get expelled with the same distribution of final velocities as for the orbits that are not captured (this works only for very hard binaries).

\subsubsection{Discussion}

We can now explain why a hard binary hardens at a constant rate that is independent of its mass ratio. The explanation given by Roos (1981) is incorrect. It implies that the dominant contribution to the hardening for a binary with $m_{1} \gg m_{2}$ comes from orbits that have close encounters with $m_{2}$ and are expelled with high velocity. But the scattering experiments show that the dominant contribution comes from orbits that do not have close encounters with the BHs and that are expelled with a velocity $v_{f} \simeq w$.

Consider a typical orbit that starts with a low velocity $v$, passes at a distance $r \simeq a$ from the two BHs, and leaves with a gain to its kinetic energy. The energy gain results mainly from the interaction with the smaller $\mathrm{BH}$ if $m_{1} \gg$ $m_{2}$ because the larger $\mathrm{BH}$ acts as a fixed potential. The interaction force of magnitude $F \sim G m_{2} / a^{2}$ acts for a time $\Delta t \sim\left(a^{3} / G M_{12}\right)^{1 / 2}$ to produce a velocity change $\Delta v \sim$ $F \Delta t \sim\left(m_{2} / M_{12}\right) V_{\text {bin }}$ and a corresponding energy change $\Delta E_{*} \sim V_{\mathrm{bin}} \Delta v \sim\left(m_{2} / M_{12}\right) V_{\mathrm{bin}}^{2}$. That gives $C \sim m_{2} / \mu \sim$ 1 , which is sufficient to give a hardening rate $H_{1}$ with no dependence on the hardness and almost no dependence on the mass ratio.

If this same derivation is repeated for a high-velocity star $\left(v>V_{\text {bin }}\right)$ it gives a hardening rate that rises as $H_{1} \sim v^{2}$ instead of falling as $H_{1} \sim 1 / v^{2}$ as it should. That is because the derivation ignores the orbits that lose energy, which tend to cancel the ones that gain energy. The cancellation removes four powers of $v$ and is the reason the hardening rate is so difficult to measure at large $v$ by the Monte Carlo method. For a hard binary there is no cancellation because the orbits that lose energy in the first encounter are captured and eventually expelled with an energy gain. It is not surprising then that the hard/nothard transition occurs at the velocity $w$ where the binary begins capturing stars effectively.

\subsection{Mass ejection}

To measure the ejection rate we need an ejection criterion, i.e. a velocity $v_{\text {ej }}$ such that a star with initial velocity $v$ is counted as ejected if it is expelled with final velocity $v_{\mathrm{f}}>$ $v_{\text {ej. }}$. The conventional escape-velocity choice, $v_{\text {ej }}=2 \sqrt{3} \sigma$, leads to a problem for a Maxwellian distribution because $0.7 \%$ of the stars have initial velocities $v>v_{\mathrm{ej}}$ and will be counted as ejected if they receive any energy from the binary, no matter how little. We therefore choose $v_{\mathrm{ej}}=$ $\max \{1.5 v, 2 \sqrt{3} \sigma\}$; the results do not depend sensitively on the numbers 1.5 and $2 \sqrt{3}$. Let $F_{\mathrm{ej}}(x, v, \sigma)$ be the fraction of stars incident upon the binary with impact parameter $x$ and initial velocity $v$ that satisfy this criterion. The ejection rate is then

$J=\frac{1}{H} \int_{0}^{\infty} d v 4 \pi v^{2} f(v, \sigma) \frac{\sigma}{v} 4 \pi \int_{0}^{\infty} d x x F_{\mathrm{ej}}(x, v, \sigma)$.

The integral over the velocity distribution is evaluated numerically after the inner integral is determined from finalvelocity distributions like those shown in Figure 1 .

The ejection rate is plotted as a function of $\sigma / V_{\mathrm{bin}}$ for five mass ratios in Figure 5. At low velocity $J$ rises as $\ln (1 / \sigma)$; at high velocity $J$ falls, first gradually, then precipitously. The velocity dependence is fit by the function

$J=j_{1}\left[1+\left(\frac{v}{j_{2} V_{\mathrm{bin}}}\right)^{j_{3}}\right]^{-j_{4}} \ln \left[1+\left(\frac{j_{2} V_{\mathrm{bin}}}{v}\right)^{j_{5}}\right]$

the five parameters are listed in Table 3. The parameters give a close fit to the data in the figure but are erratic. Note that the velocity at the bend in the curves in panel (b) is not fit well by $j_{2} V_{\text {bin }}$.

The ejection rate for a hard binary can be estimated by noting that close encounters with the binary give a mean 

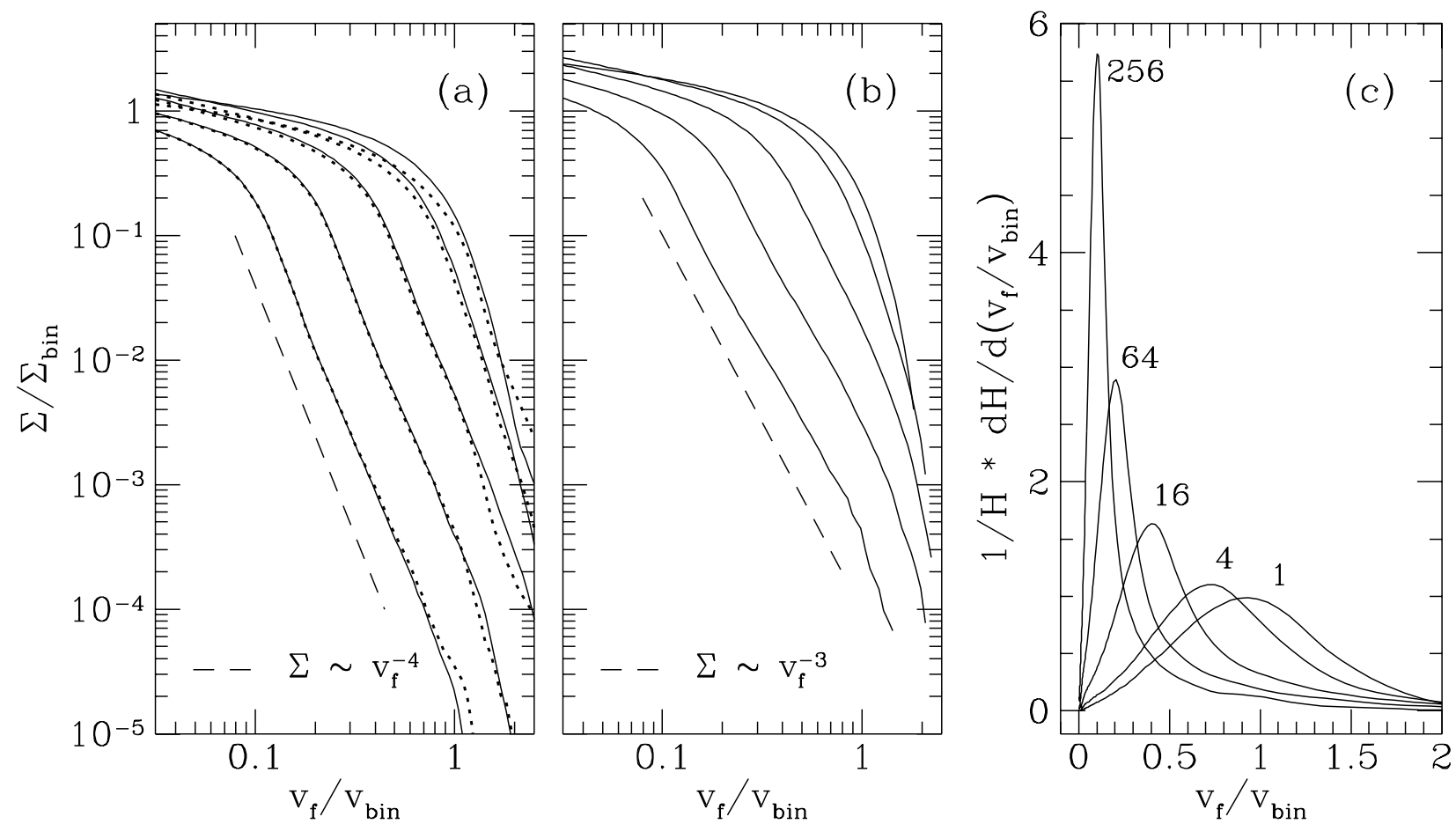

Figure 4. Distribution of final velocities for a hard, circular binary ( $v$ equals the lowest value in Fig. 1 for each mass ratio). $\Sigma$ is the cross section for an orbit to leave with velocity $\geq v_{\mathrm{f}}$ (solid lines), or to remain bound with energy $\leq-v_{\mathrm{f}}^{2} / 2$ (dotted lines). In (a) the orbits encounter the binary only once; in (b) as many times as necessary for them to leave with positive energy. Panel (c) shows the differential hardening rate for the experiments in (b). The mass ratios $m_{1} / m_{2}$ in (a) and (b) are as shown in (c), increasing from right to left.

\begin{tabular}{rrrrrr}
\hline$m_{1} / m_{2}$ & $j_{1}$ & $j_{2}$ & $j_{3}$ & $j_{4}$ & $j_{5}$ \\
\hline 1 & 0.3779 & 0.9200 & 2.2572 & 22.415 & 0.3437 \\
4 & 0.1148 & 0.8815 & 1.5224 & 10.521 & 1.4162 \\
16 & 0.0284 & 0.6608 & 0.9404 & 6.7223 & 5.6247 \\
64 & 0.0665 & 0.4438 & 0.8480 & 8.1901 & 2.1824 \\
256 & 0.2800 & 0.0214 & 3.1294 & 0.5284 & 0.8108 \\
\hline
\end{tabular}

Table 3. Parameters for fits to $J$ (eq. 24) for a circular binary.

energy change of $\langle C\rangle \simeq 1$. It then follows from the definition (8) of $C$ that a binary must interact with about its own mass in stars to shrink by a factor of $e$. But "interact with" does not mean the same as "eject." A binary that is not hard interacts with many stars but ejects few of them. And even a hard binary need not eject its own mass to shrink by a factor of $e$ if it gives some stars much more energy than others.

Figure (4) shows that a hard binary with $m_{1} / m_{2} \gg 1$ expels few stars with $v_{\mathrm{f}}>V_{\text {bin }}$. So why does $J$ not decrease rapidly with $m_{1} / m_{2}$ in the left half of Figure (5)? Because although the fraction of stars expelled with $v_{\mathrm{f}}>V_{\text {bin }}$ does decrease rapidly with $m_{1} / m_{2}$, the fraction expelled with $v_{\mathrm{f}}>w$ does not, and it is that fraction that determines the ejection rate when the binary first becomes hard.

\subsection{Eccentricity growth}

$K_{1}$ is more difficult to measure than $H_{1}$ because of the cancellation that occurs in the numerator of equation (14). The $B-C$ distribution is wide and nearly centered on the origin with a mean $\langle B-C\rangle$ that is $10-100$ times smaller than the deviation about the mean. Consequently we know much less about eccentricity growth than we know about hardening. Roos (1981) tried to measure $K_{1}$ with only 500 orbits per measurement. He found $K_{1}=0.2 \pm 0.2$ for a hard binary with $e=0.6$ and concluded that the eccentricity could increase. Mikkola and Valtonen (1992) used $10^{4}$ orbits per measurement to study the dependence of $K_{1}$ on the eccentricity and hardness of an equal-mass binary. Their results are accurate for hard binaries, for which they found positive growth rates with a maximum of $K_{1}=0.19 \pm 0.04$, but have large error bars for $v \simeq V_{\text {bin }}$.

The results derived here use $10-100$ times more orbits per measurement than Mikkola and Valtonen used $\left(10^{5}\right.$ per measurement at low stellar velocity, $10^{6}$ at high velocity) and use quasi-random numbers rather than random numbers to further reduce the statistical errors. The large number of orbits required has two practical consequences. First, only a small number of velocities and mass ratios can be examined. Second, the results should be applied only to problems where $m_{*}$ is much smaller than $m_{1}$ and $m_{2}$, for 

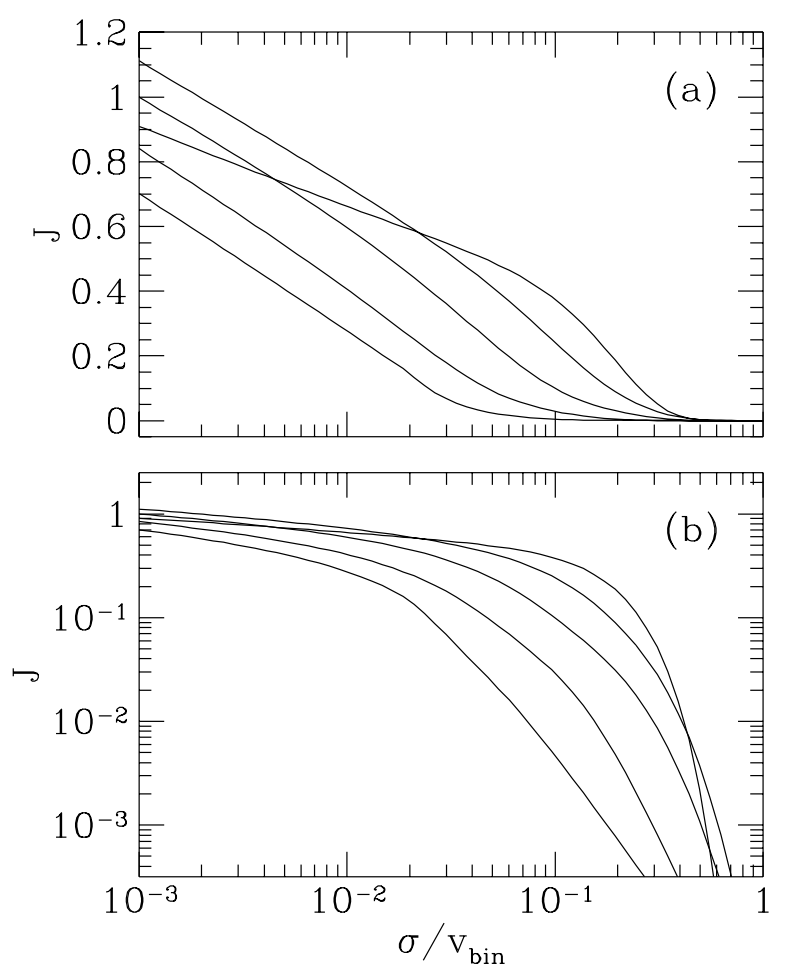

Figure 5. Mass ejection rate $J$ for a circular binary, plotted on (a) linear and (b) logarithmic scales. The five lines are for mass ratios (increasing from right to left) $m_{1} / m_{2}=1,4,16,64$, and 256.

\begin{tabular}{lrrrrrr}
\hline$v / V_{\text {bin }}$ & \multicolumn{3}{c}{$m_{1} / m_{2}=1$} & \multicolumn{3}{c}{$m_{1} / m_{2}=16$} \\
\hline & $k_{0}$ & $k_{1}$ & $k_{2}$ & $k_{0}$ & $k_{1}$ & $k_{2}$ \\
\hline 0.01 & 0.731 & 0.265 & 0.230 & 0.822 & 0.383 & 0.402 \\
0.032 & 0.841 & 0.106 & 0.534 & 0.584 & 0.552 & -0.140 \\
0.1 & 0.724 & 0.053 & 0.275 & 0.381 & 0.202 & -0.120 \\
0.32 & 1.271 & -0.198 & 0.445 & 0.739 & -0.156 & 0.135 \\
1.00 & 1.169 & 0.102 & -0.022 & 1.221 & -0.180 & 0.463 \\
\hline
\end{tabular}

Table 4. Parameters for fits to $K_{1}$ (eq. 25).

otherwise the mean behavior can get lost in the dispersion about the mean (as often happens in N-body experiments).

The measurements of $K_{1}$ are plotted in Figure 6 for five initial velocities and two mass ratios. The eccentricity dependence for each choice of $v$ and $m_{1} / m_{2}$ is fit by the function

$K_{1}(e)=e\left(1-e^{2}\right)^{k_{0}}\left(k_{1}+k_{2} e\right) ;$

the three parameters are listed in Table $1 . K_{1}$ must approach zero in the limits $e=0$ and $e=1$ : the former because of the conserved Jacobi constant; the latter because of the $\left(1-e^{2}\right)$ in equation (14).

Consider first the results for an equal-mass binary. At large stellar velocity $K_{1}$ is small and is negative for some eccentricities. As the velocity is lowered $K_{1}$ rises and becomes positive for all eccentricities. By $v / V_{\text {bin }}=0.032, K_{1}$
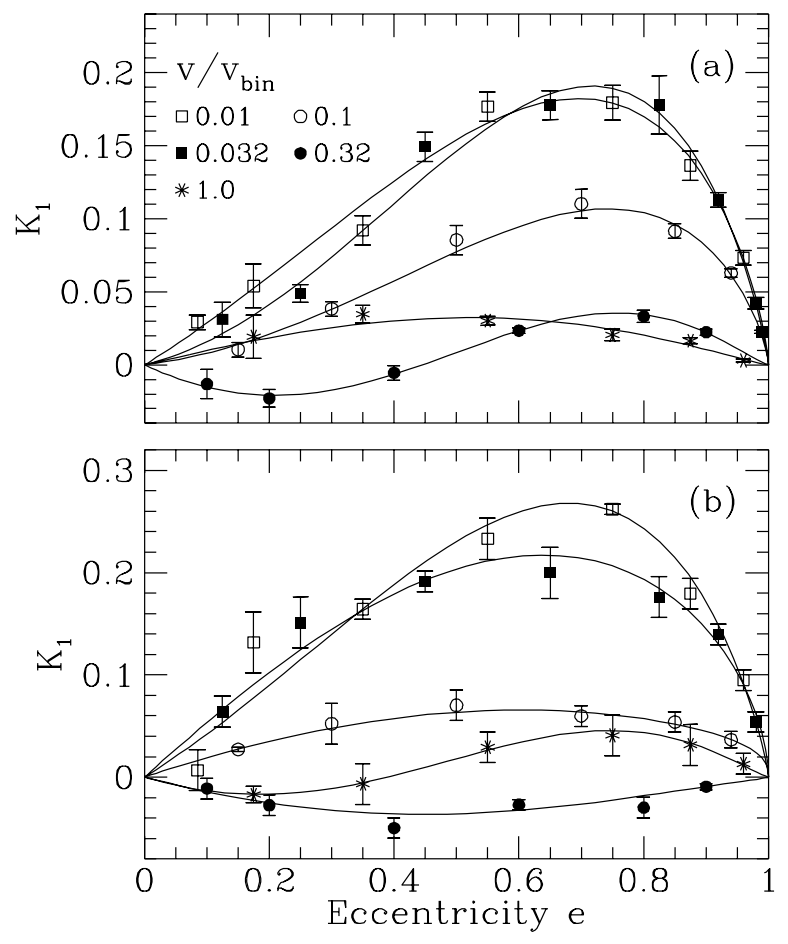

Figure 6. Eccentricity growth rate for five initial stellar velocities $v$, for binary mass ratios $m_{1} / m_{2}=1$ (a) and 16 (b). The lines are the fits to eq. (25).

has converged to its limit for a hard binary. That limit gives a maximum growth rate of $K_{1} \simeq 0.2$ near $e=0.7$, consistent with the results of Mikkola and Valtonen (1992). The main difference between these results and theirs is at high velocity, where they did not identify negative values for $K_{1}$. Those values can reduce a binary's eccentricity when it first starts to harden.

The results for $m_{1} / m_{2}=16$ differ from those for an equal-mass binary in two ways: the maximum $K_{1}$ is about $40 \%$ larger, and the hard-binary limit is reached at a lower stellar velocity. In later applications we will need the growth rate for other mass ratios. We get that by assuming that the velocity $w$ (eq. 17) determines the transition to the hard-binary limit and that this is the only important dependence on the mass ratio, i.e. by interpolating the results for $m_{1} / m_{2}=16$ at the appropriate value of $v / w$.

The derivation of the eccentricity growth rate for a Maxwellian distribution is more cumbersome than it was for the hardening rate; it is given in an appendix. The resulting $K$ is the same as $K_{1}$ at low stellar velocity but does not fall to zero as fast as $K_{1}$ at high velocity. The appendix also derives $K$ from Chandrasekhar's dynamicalfriction formula and shows that it greatly overestimates the true growth rate.

\section{APPLICATION OF RESULTS TO MASSIVE BLACK HOLE BINARIES}




\subsection{Introduction}

We consider a galaxy core with uniform density $\rho$ and velocity dispersion $\sigma$. The core mass and radius, if needed, are computed from

$M_{\mathrm{c}}=\frac{4 \pi}{3} \rho r_{\mathrm{c}}^{3}, \quad r_{\mathrm{c}}=\left(\frac{9 \sigma^{2}}{4 \pi G \rho}\right)^{1 / 2}$

Model I, for a large galaxy, has $\rho=10^{3}, \sigma=300, M_{\mathrm{c}}=$ $7.7 \times 10^{9}$, and $r_{\mathrm{c}}=120$ (dimensional quantities are given in units of $M_{\odot}, \mathrm{pc}, \mathrm{yr}$, and $\left.\mathrm{km} / \mathrm{s}\right)$. Model II, for a small, high-density galaxy, has $\rho=10^{6}, \sigma=100, M_{\mathrm{c}}=9.0 \times 10^{6}$, and $r_{\mathrm{c}}=1.3$. The BHs are assumed to enter the core and form a binary with initial eccentricity $e_{0}$ and semimajor axis $a_{0}$, the latter chosen so that $V_{\text {bin }}=\sigma / 5$. That is when the integration starts.

The equations for $d a / d t$ and $d e / d t$ for three-body scattering are combined with those of Peters (1964) for gravitational radiation:

$$
\begin{aligned}
& \left(\frac{d a}{d t}\right)_{\mathrm{gr}}=-\frac{64}{5} \frac{G^{3} m_{1} m_{2} M_{12}}{c^{5} a^{3}\left(1-e^{2}\right)^{7 / 2}}\left(1+\frac{73}{24} e^{2}+\frac{37}{96} e^{4}\right), \\
& \left(\frac{d e}{d t}\right)_{\mathrm{gr}}=-\frac{304}{15} \frac{G^{3} m_{1} m_{2} M_{12}}{c^{5} a^{4}\left(1-e^{2}\right)^{5 / 2}} e\left(1+\frac{121}{304} e^{2}\right) .
\end{aligned}
$$

The equation for $d M_{\mathrm{ej}} / d t$ is integrated to give the ejected mass but is ignored by the other equations, i.e. the density and velocity dispersion are held fixed.

\subsection{Characteristic length and time scales}

It is helpful to first consider how the length and time scales for the evolution vary with $\rho, \sigma$, and the $\mathrm{BH}$ masses (see also BBR). The binary forms at a separation $a_{\mathrm{b}}=$ $r_{\mathrm{c}}\left(M_{12} / M_{\mathrm{c}}\right)^{1 / 3}$ where the enclosed stellar mass equals $M_{12}$. It does not become hard until $w>\sqrt{3} \sigma$, which happens at about

$a_{\mathrm{h}}=\frac{G m_{2}}{4 \sigma^{2}}=1.2 \mathrm{pc}\left(\frac{m_{2}}{10^{8}}\right)\left(\frac{300}{\sigma}\right)^{2}$.

From then the binary hardens in the time

$$
\begin{aligned}
t_{\mathrm{h}} & =\left|\frac{a}{\dot{a}}\right|=\frac{\sigma}{G \rho a H} \\
& =4.3 \times 10^{7} \mathrm{yr}\left(\frac{\sigma}{300}\right)\left(\frac{10^{3}}{\rho}\right)\left(\frac{0.1}{a}\right)\left(\frac{16}{H}\right),
\end{aligned}
$$

where $H \simeq 16$ is a typical hardening rate for a hard binary (recall that $H$ is $\sqrt{2 / \pi}$ times smaller than $H_{1}$ ). This should be compared with the time for a circular binary to merge through the emission of gravitational radiation:

$$
\begin{aligned}
t_{\mathrm{gr}} & =\frac{5}{256} \frac{c^{5} a^{4}}{G^{3} \mu M_{12}^{2}} \\
& =2.9 \times 10^{6} \mathrm{yr}\left(\frac{a}{0.01}\right)^{4}\left(\frac{10^{8}}{m_{1}}\right)^{3}\left(\frac{m_{1}}{m_{2}}\right)\left(\frac{2 m_{1}}{M_{12}}\right) .
\end{aligned}
$$

The two are equal at the semimajor axis

$a_{\mathrm{gr}}=\left(\frac{256}{5} \frac{G^{2} \mu M_{12}^{2} \sigma}{c^{5} \rho H}\right)^{1 / 5}=0.027 \mathrm{pc}\left[\left(\frac{m_{1}}{10^{8}}\right)^{3}\left(\frac{m_{2}}{m_{1}}\right)\right.$

$$
\left.\left(\frac{M_{12}}{2 m_{1}}\right)\left(\frac{\sigma}{300}\right)\left(\frac{10^{3}}{\rho}\right)\left(\frac{16}{H}\right)\right]^{1 / 5} .
$$

The binary orbital velocity at $a_{\mathrm{gr}}$ is close to the geometric mean of the velocity dispersion and the speed of light:

$$
\begin{aligned}
v_{\mathrm{gr}} & =\sqrt{\frac{G M_{12}}{a_{\mathrm{gr}}}} \\
& =\sqrt{c \sigma}\left[\frac{405}{16 \pi}\left(\frac{H}{16}\right)\left(\frac{M_{12}}{M_{\mathrm{c}}}\right)^{2}\left(\frac{M_{12}}{4 \mu}\right)\right]^{1 / 10},
\end{aligned}
$$

This tells us the hardness at $a_{\mathrm{gr}}$, what we previously called $\sigma / V_{\text {bin }}$ :

$\frac{\sigma}{v_{\mathrm{gr}}} \simeq \sqrt{\frac{\sigma}{c}}=0.032\left(\frac{\sigma}{300}\right)^{1 / 2}$.

Two things should be noted about the length scales. First, $a_{\mathrm{gr}}$ is large enough that tidal disruptions cannot change the hardening rate by much unless the $\mathrm{BHs}$ are very small. The disruption radius about the larger $\mathrm{BH}$ for a star like our Sun is

$r_{\mathrm{t}}=R_{*}\left(\frac{m_{1}}{m_{*}}\right)^{1 / 3}=1.0 \times 10^{-5} \mathrm{pc}\left(\frac{R_{*}}{R_{\odot}}\right)\left(\frac{m_{1}}{10^{8} m_{*}}\right)^{1 / 3}$,

which gives $r_{\mathrm{t}} / a_{\mathrm{gr}} \simeq 4.4 \times 10^{-4}$ for the numbers in equation (32). That is too small to matter because the main contribution to hardening comes from orbits that do not have close encounters with the BH. Accretion of the disrupted stars will not matter either (although accretion from some larger gas reservoir can make a difference when the BHs are close). Tidal disruptions by the smaller BH can suppress the high-velocity ejections if $m_{1} / m_{2} \gg 1$, but those ejections do not contribute much to the hardening.

The second thing to note is the ratio $a_{\mathrm{h}} / a_{\mathrm{gr}}$, which determines how many $e$-foldings the binary has to harden between the time it becomes hard and the time radiation takes over:

$$
\begin{aligned}
\frac{a_{\mathrm{h}}}{a_{\mathrm{gr}}}=44.0 & {\left[\left(\frac{m_{1}}{10^{8}}\right)^{2}\left(\frac{m_{2}}{m_{1}}\right)^{4}\left(\frac{2 m_{1}}{M_{12}}\right)\left(\frac{300}{\sigma}\right)^{11}\right.} \\
& \left.\left(\frac{\rho}{10^{3}}\right)\left(\frac{H}{16}\right)\right]^{1 / 5} .
\end{aligned}
$$

The larger $a_{\mathrm{h}} / a_{\mathrm{gr}}$, the more mass the binary ejects and the more its eccentricity grows. For this example $a_{\mathrm{h}} / a_{\mathrm{gr}} \simeq$ $\exp (3.8)$ if $m_{1}=m_{2}$, but the ratio is smaller if $m_{1}$ is small or if $m_{2} \ll m_{1}$.

\subsection{Evolution in a fixed galaxy}

The equations have been integrated for a number of mass combinations in the two galaxy models. Figure 7 shows the hardening time and the ejected mass for an initial eccentricity of $e_{0}=0.1$ (the eccentricity does not grow by much for this choice of $e_{0}$ ).

The hardening proceeds through three stages: the first ends when the binary becomes hard at $a=a_{\mathrm{h}}$, the second starts there and ends when gravitational radiation takes over at $a=a_{\mathrm{gr}}$, and the third is the final merger stage. The gradual increase in the hardening time during the first 
stage is because of the log term in equation (19). All mass combinations harden along the same diagonal line during the second stage because all hard binaries harden at the same rate. The elapsed time equals the hardening time on that diagonal. The merger time in the final stage increases as $m_{1}$ decreases. The separation between the stages varies with the masses as expected: the smaller $m_{1}$, or the smaller $m_{2} / m_{1}$, the less room the binary has to harden between $a_{\mathrm{h}}$ and $a_{\mathrm{gr}}$. Some binaries reach $a_{\mathrm{gr}}$ before they become hard.

The hardening curves in panels (a) and (b) are similar to that shown in Figure 1 of BBR. The main differences are that BBR used a constant hardening time from Chandrasekhar's dynamical-friction formula during the first stage, and assumed that all binaries become hard at $V_{\text {bin }}=\sigma$. The hardening time used here must match onto Chandrasekhar's time at a large separation, but not until the $\log$ term in equation (19) matches the usual Coulomb logarithm.

The ejected mass is never more than a few times $M_{12}$ and is less if the BHs are small or if $m_{1} / m_{2} \gg 1$. It can be estimated by $J_{\mathrm{gr}} \ln \left(a_{\mathrm{h}} / a_{\mathrm{gr}}\right) / 2$ if $a_{\mathrm{h}} / a_{\mathrm{gr}} \gg 1$. The mass ejected by a binary with $m_{1} / m_{2} \gg 1$ can be much larger than $m_{2}$ even though it is not for an equal-mass binary; so a BH of mass $m$ ejects more mass if it merges with $N$ BHs of mass $m / N$ than if it merges with another $\mathrm{BH}$ of mass $m$. The mass displaced from the center of a real galaxy that starts with a density cusp will be larger than the $M_{\mathrm{ej}}$ computed here, because some is displaced when the BHs first approach the center and some is nearly but not quite ejected.

The dependence on the initial eccentricity is shown in Figure for one of the binaries from Figure 7 . If $e_{0} \leq 0.3$ the eccentricity hardly grows before gravitational radiation drives it to zero; if $e_{0} \simeq 0.1$ the eccentricity goes down from the start. If $e_{0}>0.3$ the eccentricity grows, but not by much. This conclusion, which Mikkola and Valtonen (1992) also reached for an equal-mass binary, is true for all the binaries in Figure 7. The merger time is reduced if $e_{0}$ is large, by about a factor of 20 if $e_{0}=0.9$ for the binary in Figure 8. The reduction could be larger if the hardening stalls during the late stages. Makino et al. (1993) say their N-body experiments show that massive $\mathrm{BH}$ binaries form with large eccentricities, but those experiments use unrealistic galaxy models and atypical initial conditions. Polnarev and Rees (1994) give arguments for why the initial eccentricity should be small.

\subsection{Changes for a galaxy that is not fixed}

We can estimate when mass ejection is likely to affect the evolution by considering the mass of stars in the unperturbed galaxy that pass within a distance $r$ of a point mass $M_{12}$ at the center:

$M\left(r_{p} \leq r\right) \simeq 10 M_{\mathrm{c}}\left(\frac{r}{r_{\mathrm{c}}}\right)^{2}\left(1+\frac{M_{12}}{M_{\mathrm{c}}} \frac{r_{\mathrm{c}}}{r}\right)$.

As the binary hardens, $M_{\mathrm{ej}}$ rises and $M\left(r_{p}<a\right)$ falls. If those two masses become equal the binary will have ejected nearly all the stars that can have a close encounter with it; further hardening must then wait for new stars to diffuse
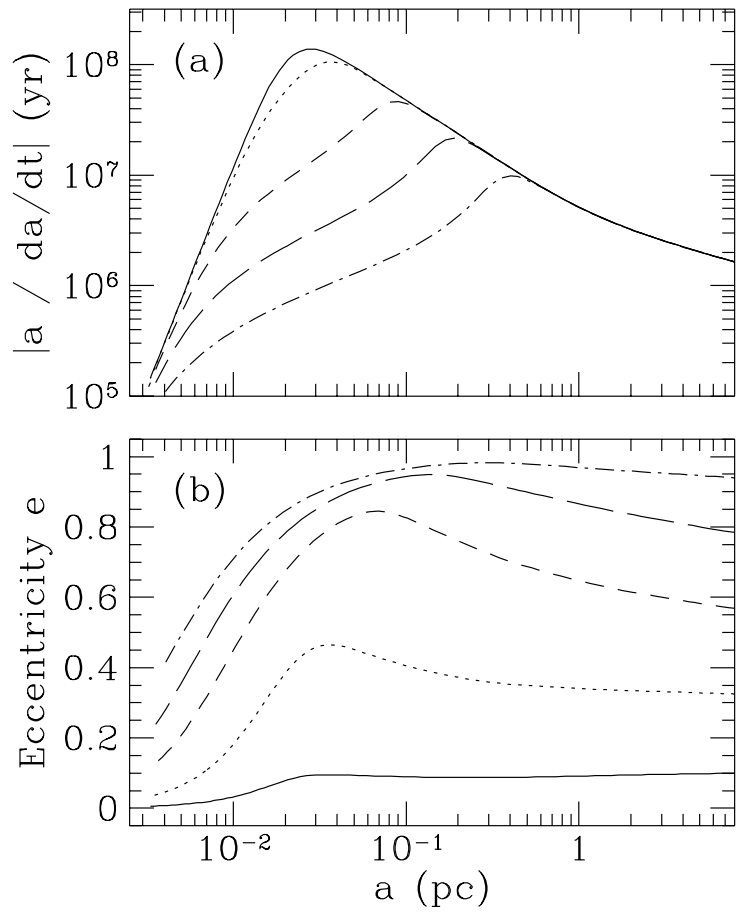

Figure 8. Eccentricity evolution. The solid line is the same as in Fig. 7 (a); the other lines assume initial eccentricities of 0.3 (dotted), 0.5, 0.7, and 0.9 (dashed-dotted).

back into the "loss-cone" orbits by two-body relaxation. The point where $M_{\mathrm{ej}}=M\left(r_{p}<a\right)$, marked by the open circles in Figure 7 , typically occurs before the binary has ejected even one tenth of the mass that it has to eject to merge. The hardening rate will be reduced by mass ejection before this point is reached. That will not change the $M_{\mathrm{ej}}(a)$ and $e(a)$ relations but it will change the time scale for $a(t)$, perhaps making the merger time longer than the age of the galaxy for some of the binaries in Figure 6 .

Mass ejection does not bring the hardening to a complete stop even in the absence of two-body relaxation because the binary does not remain fixed at $r=0$. A single particle of mass $M_{12}$ would wander from the center of the unperturbed galaxy with an amplitude

$r_{\mathrm{w}} \simeq r_{\mathrm{c}} \sqrt{\frac{m_{*}}{M_{12}}}=0.01 \mathrm{pc}\left(\frac{r_{\mathrm{c}}}{120}\right)\left(\frac{10^{8} m_{*}}{M_{12}}\right)^{1 / 2}$.

The mass scaling suggests that wandering will be more important for small BHs, but a binary with large BHs can wander too once it ejects stars from the center because there is then no restoring force to keep it fixed. The importance of wandering for the hardening rate is best studied by N-body experiments; all we can say here is that the merger times in Figure 7 are undoubtably too short.

In summary, our study of the restricted three-body problem has answered some of the questions posed at the start. It has given a complete description of how the hardening, mass ejection, and eccentricity growth depend on the properties of a massive BH binary and a uniform galaxy 

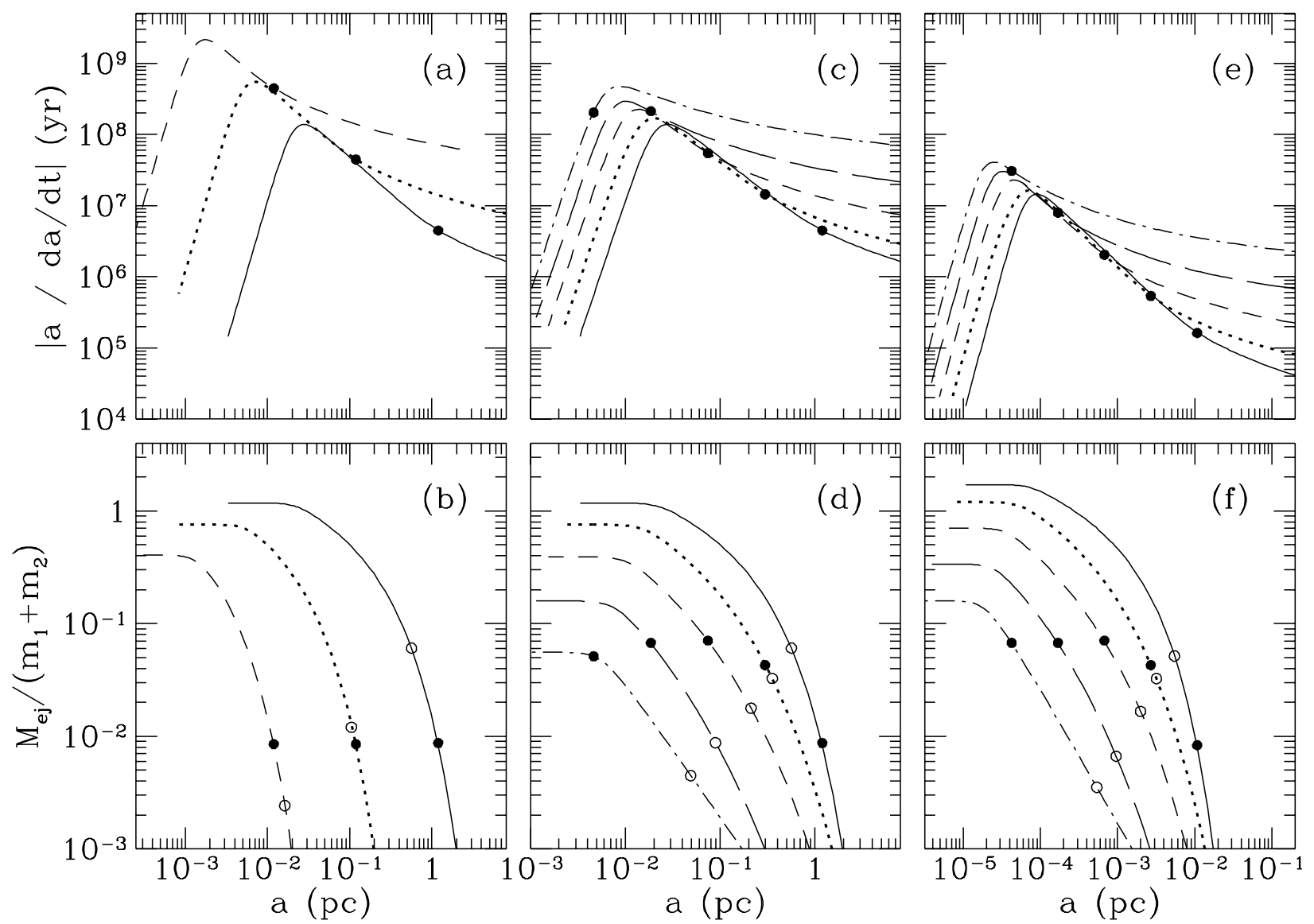

Figure 7. Hardening time $|a / \dot{a}|$ and ejected mass $M_{\mathrm{ej}}$ versus semimajor axis for BH binaries in two galaxy models: in model I (panels a-d), $\rho=10^{3}$ and $\sigma=300$; in model II (panels e and f), $\rho=10^{6}$ and $\sigma=100$. The initial eccentricity is 0.1 . In (a) and (b), $m_{1}=m_{2}=10^{8}$ (solid line), $10^{7}$ (dotted), and $10^{6}$ (dashed). In (c) and (d), $m_{1}=10^{8}$ and $m_{1} / m_{2}=1$ (solid), 4, 16, 64, and 256 (dashed-dotted). Panels (e) and (f) are similar to (c) and (d) but use galaxy model II with $m_{1}=10^{5}$. The filled and open circles mark the points where $a=a_{\mathrm{h}}$ (filled) and where the evolution could stall because of loss-cone depletion (open).

core in which it is embedded. It has cleared up some confusion resulting from mistaken applications of Chandrasekhar's dynamical-friction formula. And it has allowed us to study binary evolution in uniform galaxy cores with some simplifying assumptions. But it has left two big questions that affect the merger time: what the initial eccentricity is and by how much loss-cone depletion reduces the hardening rate. It has not allowed us to study binary evolution in realistic galaxy models with density cusps. And it has given only a crude estimate of the changes induced in such models by the evolution. These questions will be the subject of paper II.

\section{ACKNOWLEDGEMENTS}

I thank Scott Tremaine for telling me about Everhart's work, and Lars Hernquist for his encouragement and the use of his workstations. Financially support was received from NSF grant ASC 93-18185 at UCSC, and from NSF grant AST 93-18617 and NASA Theory grant NAG 5-2803 at Rutgers.

\section{REFERENCES}

Begelman, M. C., Blandford, R. D., and Rees, M. J. 1980, Massive black hole binaries in active galactic nuclei, Nature $\mathbf{2 8 7}$, 307-309.

Binney, J., and Tremaine, S. 1987, Galactic Dynamics, Princeton Univ. Press.

Chandrasekhar, S. 1944, On the stability of binary stars, Astrophys. J. 99, 54-58.

Duncan, M., Quinn, T., and Tremaine, S. 1987, The formation and extent of the solar system comet cloud, Astron. J. 94, $1330-1338$.

Ebisuzaki, T., Makino, J., and Okumura, S. K. 1991, Merging of two galaxies with central black holes, Nature 354, 212-214.

Everhart, E. 1968, Change in total energy of comets passing through the solar system, Astron. J. 73, 1039-1052.

Everhart, E. 1969, Close encounters of comets and planets, Astron. J. 73, 1039-1052. 
Everhart, E. 1976, The evolution of comet orbits, in The Study of Comets (Proc. of IAU Colloq. \#25), edited by B. Donn et al., pp. 445-464, NASA SP-393.

Fernandez, J. A. 1993, Dynamics of comets: recent developments and new challenges, in Asteroids, Comets, Meteors, edited by A. Milani, M. Di Martino, and A. Cellino, pp. 223-240, Kluwer Academic.

Fukushige, T., Ebisuzaki, T., and Makino, J. 1992, Rapid orbital decay of a black hole binary in merging galaxies, Publ. Astron. Soc. Jpn. 44, 281-289.

Gould, A. 1991, Binaries in a medium of fast low-mass objects, Astrophys. J. 379, 280-284.

Haehnelt, M. G. 1994, Low-frequency gravitational waves from supermassive black holes, Mon. Not. R. Astron. Soc. 269, 199-208.

Hairer, E., Norsett, S. P., and Wanner, G. 1987, Solving Ordinary Differential Equations I (1st ed.), Springer-Verlag.

Heggie, D. C. 1988, The scattering problem, in The Few Body Problem, edited by M. J. Valtonen, pp. 213-229, Kluwer Academic.

Hills, J. G. 1983a, The effect of low-velocity, low-mass intruders (collisionless gas) on the dynamical evolution of a binary system, Astron. J. 88, 1269-1283.

Hills, J. G. 1983b, Long-term captures of low-mass intruders by binary stars, Astron. J. 88, 1857-1867.

Hills, J. G. 1990, Encounters between single and binary stars: the effect of intruder mass on the maximum impact velocity for which the mean change in binding energy is positive, Astron. J. 99, 979-982.

Hills, J. G. 1992, Effect of intruder mass on collisions with hard binaries. II. Dependence on impact parameter and computations of the interaction cross sections, Astron. J. 103, 1955-1969.

Hut, P. 1983, Binaries as a heat source in stellar dynamics Release of binding energy, Astrophys. J. 272, L29-L33.

Hut, P. and Rees, M. J. 1992, Constraints on massive black holes as dark matter candidates, Mon. Not. R. Astron. Soc. 259, $27 \mathrm{p}-30 \mathrm{p}$.

Kormendy, J., Dressler, A., Byun, Y.-I., Faber, S. M., Grillmair, C., Lauer, T. R., Richstone, D., and Tremaine, S. 1994, HST Photometry of the cores of early-type galaxies, in ESO/OHP Workshop on Dwarf Galaxies, edited by G. Meylan and P. Prugniel, p. 147, Garching:ESO.

Makino, J., Fukushige, T., Okumura, S. K., and Ebisuzaki, T. 1993, The evolution of massive black-hole binaries in merging galaxies - I. Evolution of a binary in a spherical galaxy, Publ. Astron. Soc. Jpn. 45, 303-310.

Mikkola, S. and Valtonen, M. J. 1992, Evolution of binaries in the field of light particles and the problem of two black holes, Mon. Not. R. Astron. Soc. 259, 115-120.

Peters, P. C. 1964, Gravitational radiation and the motion of two point masses, Phys. Rev. B136, 1224-1232.

Pineault, S. and Duquet, J.-R. 1993, Binary capture of small bodies by three-body interactions and impact on to compact objects, Mon. Not. R. Astron. Soc. 261, 246-262.

Polnarev, A. G. and Rees, M. J. 1994, Binary black hole in a dense star cluster, Astron. Astrophys. 283, 301-312.

Press, W. H., Teukolsky, S. A., Vetterling, W. T., and Flannery, B. P. 1992, Numerical Recipes (Fortran, 2nd ed.), Cambridge Univ. Press.

Quinn, T., Tremaine, S., and Duncan, M. 1990, Planetary perturbations and the origin of short-period comets, Astrophys. J. 355, 667-679.

Roos, N. 1981, Galaxy mergers and active galactic nuclei, Astron. Astrophys. 104, 218-228.
Spitzer, L. 1987, Dynamical Evolution of Globular Clusters, Princeton Univ. Press.

Thorne, K. S. 1992, Sources of gravitational waves and prospects for their detection, in Recent Advances in General Relativity, edited by A. I. Janis and J. R. Porter, pp. 196-229, Birkhäuser.

Xu, G. and Ostriker, J. P. 1994, Dynamics of massive black holes as a possible candidate of galactic dark matter, Astrophys. J. 437, 184-193.

Yabushita, S. 1979, A statistical study of the evolution of the orbits of long-period comets, Mon. Not. R. Astron. Soc. $\mathbf{1 8 7}, 445-462$.

\section{APPENDIX A: ECCENTRICITY GROWTH RATE FOR A MAXWELLIAN DISTRIBUTION}

The derivation of $K$ from $K_{1}$ is more cumbersome than the derivation of $H$ from $H_{1}$ because $K_{1}$ is a function of two variables ( $e$ and $v$ ), because we do not have as much information on its velocity dependence, and because the energy and angular momentum changes in equation (12) must be averaged separately. We proceed as follows. We ignore the eccentricity dependence of $I_{x}(C)$, which we know is small, and use formula 16 for the velocity dependence. The value of $I_{x}(B)$ is found at any eccentricity and velocity from the assumed $I_{x}(C)$ and from linear interpolation of the measured $K_{1}(e, v)$. The quantities $I_{x}(C) / v$ and $I_{x}(B) / v$ are averaged over the Maxwellian distribution and substituted into equation (14) to give the growth rate $K$. There is some arbitrariness at high velocity, where we set $K_{1}(e, v)=0$ if $v>2 V_{\text {bin }}$, but that does not matter for our applications.

The growth rate was computed in this way at 13 values of $\sigma / V_{\text {bin }}$ and fit by the function (25). The parameters for the fits are plotted in panels (a), (b), and (c) of Figure A1; the resulting fits are plotted in panels (d) and (e) for the two mass ratios. $K$ and $K_{1}$ are about the same for a hard binary; there is no $\sqrt{2 / \pi}$ difference as there was for $H$. $K$ does not fall to zero as fast as $K_{1}$ at large stellar velocity, and negative values are less prominent for $K$.

These results can be compared with the prediction from Chandrasekhar's dynamical-friction formula. The frictional force on a massive particle $M$ moving with velocity $V=\sqrt{2} \sigma X$ is (eq. 7-17 of Binney and Tremaine 1987)

$\frac{d \vec{V}}{d t}=-4 \pi G^{2} M \rho \ln \Lambda \frac{\vec{V}}{V^{3}}\left[\operatorname{erf}(X)-\frac{2 X}{\sqrt{\pi}} \exp \left(-X^{2}\right)\right]$.

Consider an equal-mass binary in units where $G=M_{12}=$ $a=1$. We ignore the factor of $4 \pi G^{2} M \rho \ln \Lambda$ because it cancels in the ratio of the energy and angular momentum changes. Those changes are found by integrating over the unperturbed orbit (we use $V$ here for the relative velocity of the two BHs):

$\frac{\Delta E}{E} \propto \int_{0}^{2 \pi} d t 2 V^{2} F(V), \quad \frac{\Delta L}{L} \propto-\int_{0}^{2 \pi} d t F(V)$,

where

$F(V)=\frac{8}{V^{3}}\left[\operatorname{erf}(X / 2)-\frac{X}{\sqrt{\pi}} \exp \left(-X^{2} / 4\right)\right]$. 

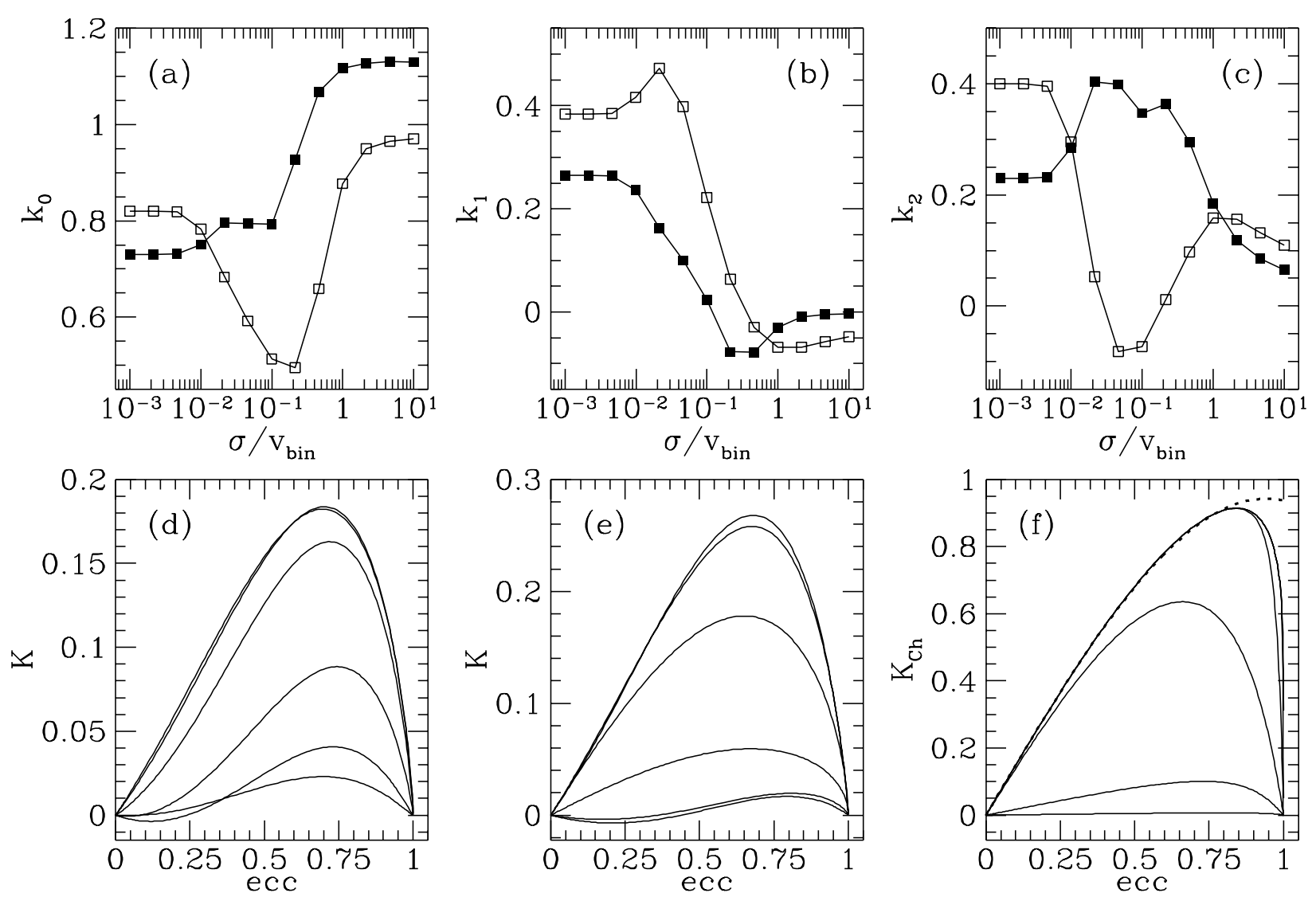

Figure A1. Eccentricity growth rate $K$ for a Maxwellian distribution. The top three panels show the fitting parameters (eq.25) for $m_{1} / m_{2}=1$ (filled squares) and 16 (open squares). The bottom three panels show the fit for $m_{1} / m_{2}=1$ (d) and 16 (e), and the prediction (for $m_{1} / m_{2}=1$ ) from Chandrasekhar's dynamical-friction formula (f); the six lines are for (from top to bottom) $\sigma / V_{\text {bin }}=0.0025,0.01,0.04,0.16,0.64$, and 2.56 (the top two lines are not distinguishable in (f)). The dotted line in (f) is the cubic approximation (A4).

The integrals are evaluated numerically and substituted into equation 12 to give the growth rate $K_{\mathrm{Ch}}$. Perturbation theory shows that for a hard binary

$K_{\mathrm{Ch}}=\frac{3}{2} e\left[1-\frac{3}{8} e^{2}+O\left(e^{4}\right)\right] \quad(X \gg 1)$,

which serves as a check on the numerical integration.

The resulting growth rate is plotted in panel (f). It differs from the correct growth rate in four ways: it falls to zero too fast as $\sigma$ rises; it rises to the hard-binary limit too fast as $\sigma$ falls; it approaches zero too slow as $e$ approaches unity; and its maximum value for a hard binary is about five times too large. The last three differences together would greatly reduce the merger time for massive $\mathrm{BH}$ binaries if $K_{\mathrm{Ch}}$ were the correct growth rate. But it isn't. 